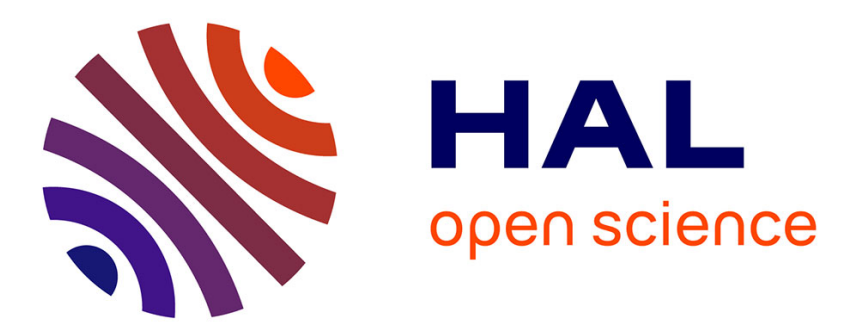

\title{
Derivation and well-posedness of the extended Green-Naghdi equations for flat bottoms with surface tension
}

\author{
Bashar Khorbatly, Ibtissam Zaiter, Samer Israwi
}

\section{To cite this version:}

Bashar Khorbatly, Ibtissam Zaiter, Samer Israwi. Derivation and well-posedness of the extended Green-Naghdi equations for flat bottoms with surface tension. Journal of Mathematical Physics, 2018, 59 (7), pp.071501. 10.1063/1.5020601 . hal-02202795

\section{HAL Id: hal-02202795 \\ https://hal.science/hal-02202795}

Submitted on 31 Jul 2019

HAL is a multi-disciplinary open access archive for the deposit and dissemination of scientific research documents, whether they are published or not. The documents may come from teaching and research institutions in France or abroad, or from public or private research centers.
L'archive ouverte pluridisciplinaire HAL, est destinée au dépôt et à la diffusion de documents scientifiques de niveau recherche, publiés ou non, émanant des établissements d'enseignement et de recherche français ou étrangers, des laboratoires publics ou privés. 


\section{Derivation and well-posedness of the extended Green-Naghdi equations for flat bottoms with surface tension}

Cite as: J. Math. Phys. 59, 071501 (2018); https://doi.org/10.1063/1.5020601

Submitted: 24 December 2017 . Accepted: 11 June 2018 . Published Online: 02 July 2018

Bashar Khorbatly (D), Ibtissam Zaiter (D), and Samer Isrwai
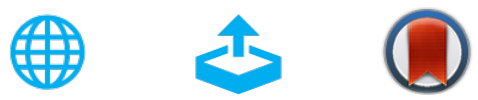

View Online

Export Citation

\section{ARTICLES YOU MAY BE INTERESTED IN}

Linear integral equations, infinite matrices, and soliton hierarchies

Journal of Mathematical Physics 59, 071101 (2018); https://

doi.org/10.1063/1.5046684

Global strong solutions for the three-dimensional Hasegawa-Mima model with partial dissipation

Journal of Mathematical Physics 59, 071503 (2018); https://

doi.org/10.1063/1.5022099

Zero resonances for localised potentials

Journal of Mathematical Physics 59, 071502 (2018); https://

doi.org/10.1063/1.5027717

Where in the world is AIP Publishing?

Find out where we are exhibiting next 


\title{
Derivation and well-posedness of the extended Green-Naghdi equations for flat bottoms with surface tension
}

\author{
Bashar Khorbatly, ${ }^{1,2, a)}$ Ibtissam Zaiter, ${ }^{2, b}$ and Samer Isrwai ${ }^{2, c)}$ \\ ${ }^{1}$ Laboratoire de Mathématique et Physique Théorique, U.F.R Sciences et Techniques Université \\ de Tours, Parc Grandmont, 37200 Tours, France \\ ${ }^{2}$ Laboratory of Mathematics-EDST, Department of Mathematics, Faculty of Sciences 1, \\ Lebanese University, Beirut, Lebanon
}

(Received 24 December 2017; accepted 11 June 2018; published online 2 July 2018)

In this paper, we will derive the two-dimensional extended Green-Naghdi system \{see Matsuno [Proc. R. Soc. A 472, 20160127 (2016)] for determination in a various way $\}$ for flat bottoms of order three with respect to the shallowness parameter $\mu$. Then we consider the $1 D$ extended Green-Naghdi equations taking into account the effect of small surface tension. We show that the construction of solution with a standard Picard iterative scheme can be accomplished in which the well-posedness in $X^{s}=H^{s+2}(\mathbb{R}) \times H^{s+2}(\mathbb{R})$ for some $s>\frac{3}{2}$ of the new extended $1 D$ system for a finite large time existence $t=O\left(\frac{1}{\varepsilon}\right)$ is demonstrated. Published by AIP Publishing. https://doi.org/10.1063/1.5020601

\section{INTRODUCTION}

The water-wave problem in its simplest form concerns two-dimensional motion of an irrotational and incompressible inviscid liquid with a free surface, acted on only by gravity and surface tension. Assume that the fluid is of constant density $\rho$ and denoted by $\Omega_{t}=\left\{(X, z) \in \mathbb{R}^{d} \times \mathbb{R},-h_{0}+b(X)<\right.$ $z<\zeta(t, X)\}$, the domain $(d=1,2)$ of the fluid for each time variable $t$ where the surface of the fluid is a graph parametrized by $\zeta$ and its bottom is parametrized by $-h_{0}+b(X)$ independent of time with $h_{0}$ the depth. This motion of this fluid is described by the following free surface Euler equations:

$$
\begin{cases}\partial_{t} V+\left(V \cdot \nabla_{X, z}\right) V=-g \vec{e}_{z}-\frac{1}{\rho} \nabla_{X, z} P & \text { in } \quad(X, z) \in \Omega_{t}, t \geq 0, \\ \nabla_{X, z} \cdot V=0 & \text { in } \quad(X, z) \in \Omega_{t}, t \geq 0, \\ \nabla_{X, z} \times V=0 & \text { in } \quad(X, z) \in \Omega_{t}, t \geq 0, \\ P-P_{a t m}=\sigma \kappa(\zeta) & \text { at } \quad z=\zeta(t, X), t \geq 0, \\ \partial_{t} \zeta-\sqrt{1+\left|\nabla_{X} \zeta\right|^{2} V \cdot n_{+}=0} & \text { at } \quad z=\zeta(t, X), t \geq 0, \\ V \cdot n_{-}=0 & \text { at } \quad z=-h_{0}+b(X), t \geq 0, \\ \lim _{|(X, z)| \rightarrow \infty}|\zeta(X, z)|+|V(t, X, z)|=0 & \text { in } \quad(X, z) \in \Omega_{t}, t \geq 0,\end{cases}
$$

where $V: \mathbb{R}_{+} \times \Omega_{t} \longrightarrow \mathbb{R}^{d} \times \mathbb{R}$ is the fluid velocity, $P: \mathbb{R}_{+} \times \Omega_{t} \longrightarrow \mathbb{R}$ is the fluid pressure term at point $(X, z) \in \Omega_{t}$ and instant $t \geq 0$, denoted by $P_{\text {atm }}$ the (constant) atmospheric pressure, $-\overrightarrow{g e} z$ is the gravitational field which is acting vertically downward with $g$ greater than zero and $\vec{e}_{z}$ is a unit

\footnotetext{
a)Electronic addresses: bashar-elkhorbatly@ hotmail.com and bashar.hussienelkhorbatly@etu.univ-tours.fr

b)E-mail: zibtissame@ hotmail.com

c)E-mail: s_israwi83@ hotmail.com
} 
vector in the vertical direction. The outward unit normal vector to the free surface and the outward unit normal to the lower boundary of $\Omega_{t}$ are respectively denoted by

$$
n_{+}=\frac{1}{\sqrt{1+\left|\nabla_{X} \zeta\right|^{2}}}\left(\nabla_{X} \zeta^{T}, 1\right)^{T} \quad \text { and } \quad n_{-}=\frac{1}{\sqrt{1+\left|\nabla_{X} b\right|^{2}}}\left(\nabla_{X} b^{T},-1\right)^{T}
$$

Denoting by $\sigma>0$ is the surface tension coefficient, and $\kappa(\zeta)=-\nabla \cdot\left(\frac{\nabla \zeta}{\sqrt{1+|\nabla \zeta|^{2}}}\right)$ is the mean curvature of the surface where $\nabla=\nabla_{X}=\left(\frac{\partial}{\partial_{x}}, \frac{\partial}{\partial_{y}}\right)^{T}$.

The complexity of this problem drove physicists, oceanographers, and mathematicians to derive simpler equations in specific physical regimes (see, for instance, Ref. 13), shallow water models $(\mu \ll 1)$, and deep water models $(\mu \nless 1)$. Many approximate models have thus been derived such as Boussinesq type equations (see Refs. 4, 8, 13, and 21 for a justification of this approximation) that simulated much of the wave motion issue in coastal engineering which can handle most of wave phenomena occurring in the nearshore areas (like refraction, diffraction, shoaling, frequency dispersion, and nonlinear interaction), but they cannot predict either where and when a wave breaks or, particularly, the hydrodynamic features of a breaking wave. Later on, Serre and Green-Naghdi introduced a higher order model (see Refs. 5, 10, and 13 for justification), which has since been widely used in coastal oceangraphy, ${ }^{11,12,20,22}$ since it considers the dispersive impacts ignored by the nonlinear shallow-water [or Saint-Venant request order $O(\mu)$ ] equations, and also it is currently the most well-known model for the numerical simulation of waterfront streams. The Green-Naghdi equations, (see Refs. 11, 12, and 15) which take into consideration neglected rotational effects (i.e., $0=\nabla_{X, z} \times V$ ), are significant for wind driven waves, waves riding upon a sheared current, waves near a ship, or tsunami waves approaching a shore and ensure the existence of $\varphi: \mathbb{R}_{+} \times \Omega_{t} \longrightarrow \mathbb{R}$, the velocity potential flow of the fluid such that $\nabla_{X, z} \varphi=V$ in $\Omega_{t}$. This plays a great role in writing the dimensionalized water-waves equations under Bernoulli's formulation with surface tension,

$$
\begin{cases}\mu \partial_{x}^{2} \varphi+\mu \partial_{y}^{2} \varphi+\partial_{z}^{2} \varphi=0 & \text { at } \quad-1+\beta b(X)<z<\varepsilon \zeta(t, X), \\ \partial_{z} \varphi-\mu \beta \nabla_{X} b \cdot \nabla_{X} \varphi=0 & \text { at } \quad z=-1+\beta b(X), \\ \partial_{t} \zeta-\frac{1}{\mu}\left(-\mu \varepsilon \nabla_{X} \zeta \cdot \nabla_{X} \varphi+\partial_{z} \varphi\right)=0 & \text { at } \quad z=\varepsilon \zeta(t, X), \\ \partial_{t} \varphi+\frac{1}{2}\left(\varepsilon\left|\nabla_{X} \varphi\right|^{2}+\frac{\varepsilon}{\mu}\left(\partial_{z} \varphi\right)^{2}\right)+\zeta=-\frac{1}{\mathrm{Bo}} \frac{\kappa(\varepsilon \sqrt{\mu} \zeta)}{\varepsilon \sqrt{\mu}} & \text { at } \quad z=\varepsilon \zeta(t, X),\end{cases}
$$

where the parameter $0 \leq \varepsilon \leq 1$ is often called a nonlinearity parameter, while $\mu \geq 0$ is the shallowness parameter, $0 \leq \beta \leq 1$ is the typical amplitude of the bottom deformations (the topography parameter), and Bo is the Bond number which measures the ratio of gravity forces over capillary forces, introduced by

$$
\varepsilon=\frac{a}{h_{0}}, \quad \mu=\frac{h_{0}^{2}}{\lambda^{2}}, \quad \beta=\frac{b_{0}}{h_{0}}, \quad \text { and } \quad \text { Bo }=\frac{\rho g \lambda^{2}}{\sigma},
$$

where we know that $a$ is the amplitude of the wave, $\lambda$ is the wave length of the wave, $b_{0}$ is the order of amplitude of the variations of the bottom topography, $h_{0}$ is the reference depth, $\rho$ is the density of the fluid, and $\sigma$ is the surface tension coefficient.

The dimensionless free surface Bernoulli's equations (2) can be reduced into a system where all functions are evaluated at the free surface (in $\mathbb{R}_{+} \times \mathbb{R}^{d}$ ), and it is known as the dimensionless version of Zakharov/Criag-Sulem ${ }^{9}$ formulation of the water-waves equations with surface tension,

$$
\left\{\begin{array}{l}
\partial_{t} \zeta-\frac{1}{\mu} \mathcal{G}_{\mu}[\varepsilon \zeta, \beta b] \psi=0, \\
\partial_{t} \psi+\zeta+\frac{\varepsilon}{2}|\nabla \psi|^{2}-\varepsilon \mu \frac{\left(\frac{1}{\mu} \mathcal{G}_{\mu}[\varepsilon \zeta, \beta b] \psi+\nabla(\varepsilon \zeta) \cdot \nabla \psi\right)^{2}}{2\left(1+\varepsilon^{2} \mu|\nabla \zeta|^{2}\right)}=-\frac{1}{\mathrm{Bo}} \frac{\kappa(\varepsilon \sqrt{\mu} \zeta)}{\varepsilon \sqrt{\mu}},
\end{array}\right.
$$


where $\psi: \mathbb{R}_{+} \times \mathbb{R}^{d} \longrightarrow \mathbb{R}$ is the trace of the velocity potential at the free surface

$$
\psi(t, X)=\varphi(t, X, \varepsilon \zeta(t, X))=\varphi_{\left.\right|_{z=\varepsilon \zeta}},
$$

and the Dirichlet-Neumann operator $\mathcal{G}_{\mu}[\varepsilon \zeta, \beta b] \cdot$ is defined by

$$
\mathcal{G}_{\mu}[\varepsilon \zeta, \beta b] \psi=-\mu(\varepsilon \nabla \zeta) \cdot(\nabla \varphi)_{\left.\right|_{z=\varepsilon \zeta}}+\left(\partial_{z} \varphi\right)_{\left.\right|_{z=\varepsilon \zeta}}=\sqrt{1+\mu \varepsilon^{2}|\nabla \zeta|^{2}}\left(\partial_{n} \varphi\right)_{\left.\right|_{z=\varepsilon \zeta}},
$$

with $\varphi$ solving (see Ref. 1 for accurate analysis) the boundary value problem

$$
\left\{\begin{array}{l}
\mu \partial_{x}^{2} \varphi+\mu \partial_{y}^{2} \varphi+\partial_{z}^{2} \varphi=0 \quad \text { in } \quad-1+\beta b(X)<z<\varepsilon \zeta(t, X) \\
\partial_{n} \varphi_{\left.\right|_{z=-1+\beta b}}=0 \\
\varphi_{\left.\right|_{z=\varepsilon \zeta}}=\psi(t, X)
\end{array}\right.
$$

where $\partial_{n} \varphi=n_{-} \cdot \nabla_{X, z} \varphi$ refers to the upward normal derivative at the bottom.

In the event that no presumption is made on the nonlinearity parameter defined above, a shallowwater asymptotic regime is identified assuming that the water depth is small enough with respect to the wave length. Formally, this regime at second order $O\left(\mu^{2}\right)$ leads to a large amplitude model $(\mu \ll 1, \varepsilon \sim 1)$ called the Green-Naghdi system. A rigorous justification on the well-posedness of the standard Green-Naghdi equations was given by several works such as Refs. 2, 3, and 10 in $1 D$ and $2 D$ with flat and non-flat bottoms $(\beta=0)$, respectively, where a solution was constructed with a standard Picard iterative scheme so that there is no loss of regularity of the solution with respect to the initial condition, unlike the Nash-Moser scheme made in Ref. 14 for a $2 D$ case. The aim of this paper is to derive the $2 D$ extended (of order $\mu^{3}$ ) Green-Naghdi system for flat bottoms (done by Matsuno in Refs. 6 and 7, applying the method used in Ref. 15 for $O\left(\mu^{2}\right)$ approximation with non-flat bottoms) represented by

$$
\left\{\begin{aligned}
& \partial_{t} \zeta+\nabla \cdot(h v)=0, \\
&\left(h+\mu \mathcal{T}[h]+\mu^{2} \mathfrak{T}[h]\right) \partial_{t} v+ h \nabla \zeta \\
&+\varepsilon h(v \cdot \nabla) v+\varepsilon \mu Q_{1}[U] v+\varepsilon \mu^{2} Q_{2}[U] v=O\left(\mu^{3}\right),
\end{aligned}\right.
$$

where $v=\left(v_{1}, v_{2}\right)^{T}, U=(\zeta, v)^{T}$, and $h=1+\varepsilon \zeta$, denoted by

$\mathcal{T}[h] v=-\frac{1}{3} \nabla\left(h^{3} \nabla \cdot v\right) \quad \mathfrak{T}[h] v=-\frac{1}{45} \nabla\left(\nabla \cdot\left(h^{5} \nabla(\nabla \cdot v)\right)\right) \quad Q_{1}[U] v=-\frac{1}{3} \nabla\left(h^{3}\left((v \cdot \nabla)(\nabla \cdot v)-(\nabla \cdot v)^{2}\right)\right)$

with

$$
\begin{aligned}
Q_{2}[U] v=-\frac{1}{45} \nabla[\nabla \cdot\{ & \left.\left.h^{5}\left(\nabla^{2}(\nabla \cdot v)\right) v-5 h^{5}(\nabla \cdot v) \nabla(\nabla \cdot v)+\nabla h^{5} \times(v \times \nabla(\nabla \cdot v))\right\}\right] \\
& +\frac{2}{45} \nabla\left(h^{5}(\nabla(\nabla \cdot v))^{2}\right)+\frac{1}{45} \nabla \cdot\left(h^{5} \nabla(\nabla \cdot v)\right) \nabla(\nabla \cdot v)+\frac{1}{90} h^{5} \nabla\left\{(\nabla(\nabla \cdot v))^{2}\right\} .
\end{aligned}
$$

This model which takes into account, the dispersive effects neglected by shallow-water where the existence of $\mu^{2}$ terms makes the analysis more difficult. The construction of a solution ( $1 D$ case) with a standard Picard iterative scheme as in Refs. 2 and 10 cannot be achieved without considering the effect of small surface tension that smoothes the way in controlling the energy estimates. Our objective here is to demonstrate that it is additionally conceivable to utilize such an iterative scheme to study the well-posedness of the extended $1 D$ Green-Naghdi equations of order $\mu^{3}$ with surface tension.

\section{A. Organization of the paper}

The aim of this paper is to derive and study the extended shallow-water approximation to the full water wave problem, and it is ordered as follows. First of all, in Sec. II, we derive the extended $2 D$ Green-Naghdi system for flat bottoms with or without surface tension. Then in Sec. III A, some preliminary results are given. The well-posedness of the system is stated in Sec. III B and then proved in Sec. III C. 


\section{B. Notation}

We denote by $C\left(\lambda_{1}, \lambda_{2}, \ldots\right)$ a constant depending on the parameters $\lambda_{1}, \lambda_{2}, \ldots$ and whose dependence on the $\lambda_{j}$ is always assumed to be nondecreasing.

The notation $a \lesssim b$ means that $a \leq C b$, for some nonnegative constant $C$ whose exact expression is of no importance (in particular, it is independent of the small parameters involved).

Let $p$ be any constant with $1 \leq p<\infty$ and denote $L^{p}=L^{p}\left(\mathbb{R}^{d}\right)$ the space of all Lebesgue-measurable functions $f$ with the standard norm $|f|_{L^{p}}=\left(\int_{\mathbb{R}^{d}}|f(X)|^{p} d X\right)^{1 / p}<\infty$.

When $p=2$, we denote the norm $|\cdot|_{L^{2}}$ simply by $|\cdot|_{2}$. The inner product of any functions $f_{1}$ and $f_{2}$ in the Hilbert space $L^{2}\left(\mathbb{R}^{d}\right)$ is denoted by $\left(f_{1}, f_{2}\right)=\int_{\mathbb{R}^{d}} f_{1}(X) f_{2}(X) d X$.

The space $L^{\infty}=L^{\infty}\left(\mathbb{R}^{d}\right)$ consists of all essentially bounded Lebesgue-measurable functions $f$ with the norm $|f|_{L^{\infty}}=\operatorname{ess} \sup |f(X)|<\infty$.

We denote by $W^{1, \infty}=W^{1, \infty}\left(\mathbb{R}^{d}\right)=\left\{f \in L^{\infty}, \nabla f \in\left(L^{\infty}\right)^{d}\right\}$ endowed with its canonical norm.

For any real constant $s, H^{s}=H^{s}\left(\mathbb{R}^{d}\right)$ denotes the Sobolev space of all tempered distributions $f$ with the norm $|f|_{H^{s}}=\left|\Lambda^{s} f\right|_{2}<\infty$, where $\Lambda$ is the pseudo-differential operator $\Lambda^{s}=\left(1-\partial_{x}^{2}\right)^{s / 2}$.

For any functions $u=u(X, t)$ and $v(X, t)$ defined on $\mathbb{R}^{d} \times[0, T)$ with $T>0$, we denote the inner product, the $L^{p}$-norm and especially the $L^{2}$-norm, as well as the Sobolev norm, with respect to the spatial variable $X$, by $(u, v)=(u(\cdot, t), v(\cdot, t)),|u|_{L^{p}}=|u(\cdot, t)|_{L^{p}},|u|_{L^{2}}=|u(\cdot, t)|_{L^{2}}$, and $|u|_{H^{s}}=|u(\cdot, t)|_{H^{s}}$, respectively.

Let $f(X, t)$ be a vector field defined on $\mathbb{R}^{d} \times[0, \infty)$ of the independent variable $X=\left(x_{1}, x_{2}, \ldots, x_{d}\right) \in \mathbb{R}^{d}$; its partial derivative with respect to $x_{k}$ is denoted by $\partial_{x_{k}} f=f_{x_{k}}$ for $1 \leq k$ $\leq d$. This allows us to define the gradient of $f$, and we denote it as $\nabla f=\left(\partial_{x_{1}} f_{1}, \partial_{x_{2}} f_{2}, \ldots, \partial_{x_{d}} f_{d}\right) \in \mathbb{R}^{d}$. Also we call divergence of $f$ the scalar denoted $\nabla \cdot f=\sum_{i=1}^{d} \partial_{x_{i}} f_{i}$ and when $d=3$, we call the curl of $f$ the vector denoted $\nabla \times f=\left(\partial_{x_{2}} f_{3}-\partial_{x_{3}} f_{2}, \partial_{x_{3}} f_{1}-\partial_{x_{1}} f_{3}, \partial_{x_{1}} f_{2}-\partial_{x_{2}} f_{1}\right)^{T}$.

For any closed operator $T$ defined on a Banach space $Y$ of functions, the commutator $[T, f]$ is defined by $[T, f] g=T(f g)-f T(g)$ with $f, g$, and $f g$ belonging to the domain of $T$.

\section{DERIVATION OF THE EXTENDED 2D GREEN-NAGHDI SYSTEM}

To derive the Green-Naghdi equations (the $2 D$ case), we introduce the depth averaged horizontal velocity,

$$
v(t, X)=\frac{1}{h(t, X)} \int_{-1+\beta b(X)}^{\varepsilon \zeta(t, X)} \nabla \varphi(t, X, z) d z \quad \text { with } \quad h(t, X)=1+\varepsilon \zeta(t, X)-\beta b(X) .
$$

The first equation of the Green-Naghdi system $(7), \partial_{t} \zeta+\nabla \cdot(h v)=0$ which exactly coincides with the first equation of (3) stems from a clear outcome of Green's identity or by a straightforward calculation and rearranging terms using (2). Now as in Ref. 15, in order to derive the evolution equation on $v$, the key point is to obtain an asymptotic expansion $\nabla \psi$ with respect to $\mu$ and in terms of $v$ and $\zeta$. Since $\mu \ll 1$, we look for an asymptotic expansion of $\varphi$ under the form

$$
\varphi_{a p p}(t, X, z)=\varphi_{0}+\mu \varphi_{1}+\mu^{2} \varphi_{2}+\cdots+\mu^{N} \varphi_{N}=\sum_{j=0}^{N} \mu^{j} \varphi_{j}
$$

Plugging expression (9) into the boundary value problem (6) and after dropping all terms of order $O\left(\mu^{N+1}\right)$, one gets

$$
\forall j=0,1, \ldots, N \quad \partial_{z}^{2} \varphi_{j}=-\partial_{x}^{2} \varphi_{j-1}-\partial_{y}^{2} \varphi_{j-1},
$$

with the convention $\varphi_{-1}=0$ by definition and the boundary condition

$$
\forall j=0,1, \ldots, N \quad\left\{\begin{array}{l}
-\beta \nabla b \nabla \varphi_{j-1}+\partial_{z} \varphi_{j}=0 \quad \text { at } \quad z=-1+\beta b, \\
\left(\varphi_{j}\right)_{\left.\right|_{z=\varepsilon \zeta}}=\delta_{0, j} \psi,
\end{array}\right.
$$


where $\delta_{0, j}=1$ if $j=0$ and zero otherwise. Solving the ordinary differential equation (ODE) (10) with (11) yields the following:

$$
\begin{aligned}
& \varphi_{0}(t, X, z)=\psi(t, X), \\
& \varphi_{1}(t, X, z)=(z-\varepsilon \zeta)\left(-\frac{1}{2}(z+\varepsilon \zeta)-1+\beta b\right) \nabla \cdot(\nabla \psi)+\beta(z-\varepsilon \zeta) \nabla b \cdot \nabla \psi .
\end{aligned}
$$

It is sufficient to have only $\varphi_{0}, \varphi_{1}$ in order to derive the equations to order $\mu^{2}$. But for obtaining the extended Green-Naghdi system, it is essential to find $\varphi_{2}$. By proceeding in the same way as above and with the help of (12) and (13), one gets after denoting that $w=\nabla \psi$

$$
\begin{aligned}
& \varphi_{2}(t, X, z)=(z-\varepsilon \zeta) \beta \nabla b \cdot\left(\nabla \varphi_{1}\right)_{\left.\right|_{z=-1+\beta b}}+\frac{1}{2}\left((z+1-\beta b)^{2}-h^{2}\right)(\varepsilon \nabla \zeta)(-\varepsilon \nabla \zeta+2(\beta \nabla b)) \nabla \cdot w \\
& \quad-2\left[\frac{1}{2}\left((z+1-\beta b)^{2}-h^{2}\right) h(\varepsilon \nabla \zeta)+\frac{1}{2}\left(\frac{1}{3}(z-\varepsilon \zeta)^{3}-(z-\varepsilon \zeta) h^{2}\right)(\beta \nabla b)\right] \nabla(\nabla \cdot w) \\
& -\left[\frac{1}{2}\left((z+1-\beta b)^{2}-h^{2}\right) h \nabla \cdot(\varepsilon \nabla \zeta)+\frac{1}{2}\left(\frac{1}{3}(z-\varepsilon \zeta)^{3}-(z-\varepsilon \zeta) h^{2}\right) \nabla \cdot(\beta \nabla b)\right] \nabla \cdot w \\
& +\left[\frac{1}{24}\left(z^{4}-(\varepsilon \zeta)^{4}\right)-\frac{1}{6}(-1+\beta b)^{3}(z-\varepsilon \zeta)-\frac{(\varepsilon \zeta)^{2}}{4}\left((z+1-\beta b)^{2}-h^{2}\right)\right. \\
& \left.\quad-\frac{1}{2}\left(\frac{1}{3}(z-\varepsilon \zeta)^{3}-h^{2}(z-\varepsilon \zeta)\right)(-1+\beta b)\right] \nabla \cdot(\nabla(\nabla \cdot w)) \\
& +\left((z+1-\beta b)^{2}-h^{2}\right)(\varepsilon \nabla \zeta) \nabla(\beta \nabla b \cdot w)+\frac{1}{2}\left((z+1-\beta b)^{2}-h^{2}\right) \nabla \cdot(\varepsilon \nabla \zeta) \beta \nabla b \cdot w \\
& -\frac{1}{2}\left(\frac{1}{3}(z-\varepsilon \zeta)^{3}-(z-\varepsilon \zeta) h^{2}\right) \nabla \cdot(\nabla(\beta \nabla b \cdot w)),
\end{aligned}
$$

the polynomial of order 4 in $z$. So the horizontal component of the velocity in the fluid domain is given by

$$
V(t, X, z)=\nabla \varphi_{a p p}=\nabla \varphi_{0}(t, X, z)+\mu \nabla \varphi_{1}(t, X, z)+\mu^{2} \nabla \varphi_{2}(t, X, z)+O\left(\mu^{3}\right) .
$$

The averaged velocity is thus given by

$$
v(t, X)=\nabla \psi+\frac{\mu}{h} \int_{-1+\beta b(X)}^{\varepsilon \zeta(t, X)} \nabla \varphi_{1} d z+\frac{\mu^{2}}{h} \int_{-1+\beta b(X)}^{\varepsilon \zeta(t, X)} \nabla \varphi_{2} d z+O\left(\mu^{3}\right) .
$$

For flat bottoms, we take here and throughout the rest of this paper $\beta=0$.

As in Ref. 15, we have

$$
\int_{-1}^{\varepsilon \zeta(t, X)} \nabla \varphi_{1} d z=\mathcal{T}[h] \nabla \psi=-\frac{1}{3} \nabla\left(h^{3} \nabla \cdot(\nabla \psi)\right) .
$$

In order to compute $J=\int_{-1}^{\varepsilon \zeta(t, X)} \nabla \varphi_{2} d z$, denote by $w=\nabla \psi$ (noting that $w$ is independent of $z$ ),

$$
\begin{aligned}
& \nabla \varphi_{2}(t, X, z)=(h \nabla h) h \nabla \cdot(\nabla h \nabla \cdot w)+\frac{1}{2}\left(h^{2}-(z+1)^{2}\right) \nabla(h \nabla \cdot(\nabla h \nabla \cdot w)) \\
& +h \nabla h(\nabla h \nabla(h \nabla \cdot w))+\frac{1}{2}\left(h^{2}-(z+1)^{2}\right) \nabla(\nabla h \nabla(h \nabla \cdot w)) \\
& +\left[\frac{1}{24}\left(z^{4}-(\varepsilon \zeta)^{4}\right)+\frac{1}{6}(z-\varepsilon \zeta)-\frac{(\varepsilon \zeta)^{2}}{4}\left((z+1)^{2}-h^{2}\right)\right. \\
& \left.+\frac{1}{2}\left(\frac{1}{3}(z-\varepsilon \zeta)^{3}-h^{2}(z-\varepsilon \zeta)\right)\right] \nabla\{\nabla \cdot(\nabla(\nabla \cdot w))\} \\
& +\left[-\frac{1}{6}(\varepsilon \zeta)^{3} \nabla h-h \nabla h(z-\varepsilon \zeta)-\left(\frac{1}{6}-\frac{1}{2} h^{2}\right) \nabla h-\frac{(\varepsilon \zeta)}{2} \nabla h\left((z+1)^{2}-h^{2}\right)\right. \\
& \left.+\frac{(\varepsilon \zeta)^{2}}{2} h \nabla h-\frac{1}{2}(z-\varepsilon \zeta)^{2} \nabla h\right] \nabla \cdot(\nabla(\nabla \cdot w)) \\
& =J_{1}+J_{2}+\ldots+J_{6} .
\end{aligned}
$$


Now after some calculations, we have the following integrals:

$$
\begin{aligned}
& \int_{-1}^{\varepsilon \zeta(t, X)}\left(J_{1}+J_{2}\right) d z=\frac{1}{3} \nabla\left(h^{4} \nabla \cdot(\nabla h \nabla \cdot w)\right), \\
& \int_{-1}^{\varepsilon \zeta(t, X)}\left(J_{3}+J_{4}\right) d z=\frac{1}{12} \nabla\left(\nabla h^{4} \nabla(h \nabla \cdot w)\right), \\
& \int_{-1}^{\varepsilon \zeta(t, X)}\left(J_{5}+J_{6}\right) d z=\frac{2}{15} \nabla\left(h^{5} \nabla \cdot(\nabla(\nabla \cdot w))\right),
\end{aligned}
$$

with

$\int_{-1}^{\varepsilon \zeta(t, X)}\left[\frac{1}{24}\left(z^{4}-(\varepsilon \zeta)^{4}\right)+\frac{1}{6}(z-\varepsilon \zeta)-\frac{(\varepsilon \zeta)^{2}}{4}\left((z+1)^{2}-h^{2}\right)+\frac{1}{2}\left(\frac{1}{3}(z-\varepsilon \zeta)^{3}-h^{2}(z-\varepsilon \zeta)\right)\right] d z=\frac{2}{15} h^{5}$

and

$\int_{-1}^{\varepsilon \zeta(t, X)}\left[-\frac{1}{6}(\varepsilon \zeta)^{3}-h(z-\varepsilon \zeta)-\left(\frac{1}{6}-\frac{1}{2} h^{2}\right)-\frac{(\varepsilon \zeta)}{2}\left((z+1)^{2}-h^{2}\right)+\frac{(\varepsilon \zeta)^{2}}{2} h-\frac{1}{2}(z-\varepsilon \zeta)^{2}\right] \nabla h \quad d z=\frac{2}{15} \nabla h^{5}$

Hence, we get

$$
J=\int_{-1}^{\varepsilon \zeta(t, X)} \nabla \varphi_{2} d z=\frac{2}{15} \nabla\left(\nabla \cdot\left(h^{5} \nabla(\nabla \cdot w)\right)\right)+\frac{1}{3} \nabla\left(h^{3} \nabla \cdot(h \nabla h) \nabla \cdot w\right)=\mathcal{T}^{\prime}[h] w .
$$

Thus, we have

$$
v=\nabla \psi-\frac{\mu}{h} \mathcal{T}[h] \nabla \psi+\frac{\mu^{2}}{h} \mathcal{T}^{\prime}[h] \nabla \psi+O\left(\mu^{3}\right)
$$

but

$$
\nabla \psi=v+\frac{\mu}{h} \mathcal{T}[h] v+\frac{\mu^{2}}{h}\left[\mathcal{T}[h]\left(\frac{1}{h} \mathcal{T}[h] v\right)-\mathcal{T}^{\prime}[h] v\right]
$$

and

$$
\mathcal{T}[h]\left(\frac{1}{h} \mathcal{T}[h] v\right)=\frac{1}{9} \nabla\left(\nabla \cdot\left(h^{5} \nabla(\nabla \cdot v)\right)\right)+\frac{1}{3} \nabla\left(h^{3} \nabla \cdot(h \nabla h) \nabla \cdot v\right) .
$$

Therefore, we obtain

$$
\nabla \psi=v+\frac{\mu}{h} \mathcal{T}[h] v+\frac{\mu^{2}}{h} \mathfrak{T}[h] v+O\left(\mu^{3}\right)
$$

where

$$
\mathcal{T}[h] v=-\frac{1}{3} \nabla\left(h^{3} \nabla \cdot v\right) \quad \text { and } \quad \mathfrak{T}[h] v=-\frac{1}{45} \nabla\left(\nabla \cdot\left(h^{5} \nabla(\nabla \cdot v)\right)\right) .
$$

Now, in order to derive the extended Green-Naghdi equations for flat bottoms without surface tension (i.e., $\sigma=0$ ), we will take the gradient of the second equation of (3), then multiply it by $h$, and replace $\nabla \psi$ by its expression (20) and $\frac{1}{\mu} \mathcal{G}[\varepsilon \zeta, \beta b] \psi$ by $\nabla \cdot(h v)=\nabla h \cdot v+h \nabla \cdot v$ in the resulting equations. Moreover, we drop the $O\left(\mu^{3}\right)$ terms, and we use the following vector triple products and the vector identities:

$$
\begin{gathered}
u \times(v \times \omega)=(u \cdot \omega) v-(u \cdot v) \omega, \\
\nabla \times(\nabla G)=0 \quad \text { and } \quad \nabla \times(G F)=G \nabla \times F+\nabla G \times F,
\end{gathered}
$$

where $G$ is a differentiable scalar function and $u, v, \omega, F$ are differentiable vector fields; one gets the same results obtained in Refs. 6 and 7 done by Matsuno applying another method,

$$
\left\{\begin{aligned}
& \partial_{t} \zeta+\nabla \cdot(h v)=0, \\
&\left(h+\mu \mathcal{T}[h]+\mu^{2} \mathfrak{T}[h]\right) \partial_{t} v+h \nabla \zeta \\
&+\varepsilon h(v \cdot \nabla) v+\varepsilon \mu Q_{1}[U] v+\varepsilon \mu^{2} Q_{2}[U] v=O\left(\mu^{3}\right),
\end{aligned}\right.
$$


where $v=\left(v_{1}, v_{2}\right)^{T}, U=(\zeta, v)^{T}$, and $h(t, X)=1+\varepsilon \zeta(t, X)$, denoted by

$\mathcal{T}[h] v=-\frac{1}{3} \nabla\left(h^{3} \nabla \cdot v\right) \quad \mathfrak{T}[h] v=-\frac{1}{45} \nabla\left(\nabla \cdot\left(h^{5} \nabla(\nabla \cdot v)\right)\right) \quad Q_{1}[U] v=-\frac{1}{3} \nabla\left(h^{3}\left((v \cdot \nabla)(\nabla \cdot v)-(\nabla \cdot v)^{2}\right)\right)$,

with

$$
\begin{aligned}
Q_{2}[U] v=-\frac{1}{45} \nabla[\nabla \cdot\{ & \left.\left.h^{5}\left(\nabla^{2}(\nabla \cdot v)\right) v-5 h^{5}(\nabla \cdot v) \nabla(\nabla \cdot v)+\nabla h^{5} \times(v \times \nabla(\nabla \cdot v))\right\}\right] \\
& +\frac{2}{45} \nabla\left(h^{5}(\nabla(\nabla \cdot v))^{2}\right)+\frac{1}{45} \nabla \cdot\left(h^{5} \nabla(\nabla \cdot v)\right) \nabla(\nabla \cdot v)+\frac{1}{90} h^{5} \nabla\left\{(\nabla(\nabla \cdot v))^{2}\right\},
\end{aligned}
$$

where the expression of $Q_{2}$ introduces the Laplacian operator $\nabla^{2}=\nabla \cdot \nabla=\Delta$.

In the presence of surface tension $(\sigma \neq 0)$, different strategies exist to deal with it in the waterwave problem such as Refs. 9 and 16-18; the main contrast in our work is that the gradient of the capillary term $-\frac{1}{\mathrm{Bo}} \frac{\kappa(\varepsilon \sqrt{\mu} \zeta)}{\varepsilon \sqrt{\mu}}$ multiplied by $h$ must be added to the right-hand side of the second equation in (24).

Let us define the rescaled Bond number bo instead of the classical Bond number Bo, as follows:

$$
\mathrm{bo}=\mu \mathrm{Bo}=\frac{\rho g h_{0}^{2}}{\sigma}>0,
$$

where $h_{0}$ denotes the reference depth, $\rho$ denotes the positive constant density of the fluid, $g$ denotes the acceleration of gravity, and $\sigma>0$ denotes the surface tension coefficient so that $\mathrm{Bo}^{-1}=\mu \mathrm{bo}^{-1}=$ $O(\mu)$, and the capillary term that should be added becomes

$$
-\frac{1}{\mathrm{Bo}} h \nabla\left\{\frac{\kappa(\varepsilon \sqrt{\mu} \zeta)}{\varepsilon \sqrt{\mu}}\right\}=\frac{1}{\mathrm{bo}} \mu h \nabla(\nabla \cdot(\nabla \zeta))-\frac{1}{2 \mathrm{bo}} \varepsilon^{2} \mu^{2} h \nabla\left(\nabla \cdot\left(|\nabla \zeta|^{2} \nabla \zeta\right)\right)+O\left(\varepsilon^{4} \mu^{3}\right) .
$$

\section{WELL-POSEDNESS OF THE EXTENDED 1D GREEN-NAGHDI SYSTEM WITH SURFACE TENSION}

For one-dimensional $(d=1)$ surfaces, the Green-Naghdi system (24) with surface tension can be rearranged after a few calculations, and considering (25), one may write

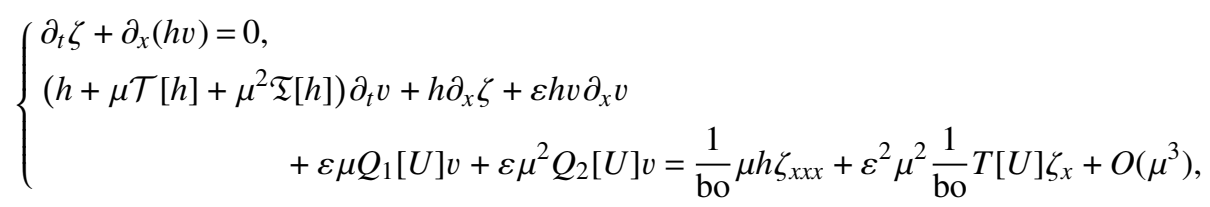

where $U=(\zeta, v)^{T}$ and $h(t, x)=1+\varepsilon \zeta(t, x)$, denoted by

$$
\mathcal{T}[h] v=-\frac{1}{3} \partial_{x}\left(h^{3} \partial_{x} v\right), \quad \mathfrak{T}[h] v=-\frac{1}{45} \partial_{x}^{2}\left(h^{5} \partial_{x}^{2} v\right), \quad Q_{1}[U] v=-\frac{1}{3} \partial_{x}\left(h^{3}\left(v v_{x x}-v_{x}^{2}\right)\right),
$$

and

$$
Q_{2}[U] v=-\frac{1}{45} \partial_{x}\left\{\partial_{x}\left(h^{5}\left(v v_{x x x}-5 v_{x} v_{x x}\right)\right)-3 h^{5}\left(v_{x x}\right)^{2}\right\}, \quad T[U] \zeta_{x}=-\frac{1}{2} h \partial_{x}^{2}\left(\zeta_{x}^{2} \zeta_{x}\right) .
$$

Now, adding and subtracting $\varepsilon \mu \mathcal{T}[h]\left(v v_{x}\right)$ and $\varepsilon \mu^{2} \mathfrak{T}[h]\left(v v_{x}\right)$ in the second equation of (26), one gets

$$
\left\{\begin{aligned}
\partial_{t} \zeta+\partial_{x}(h v) & =0, \\
(h+\mu \mathcal{T}[h] & \left.+\mu^{2} \mathfrak{T}[h]\right)\left(\partial_{t} v+\varepsilon v v_{x}\right)+h \partial_{x} \zeta+\frac{2}{3} \varepsilon \mu \partial_{x}\left(h^{3} v_{x}^{2}\right) \\
& +\frac{1}{45} \varepsilon \mu^{2} \partial_{x}\left\{8 \partial_{x}\left(h^{5} v_{x} v_{x x}\right)+3 h^{5} v_{x x}^{2}\right\}=\frac{1}{\mathrm{bo}} \mu h \zeta_{x x x}+\varepsilon^{2} \mu^{2} \frac{1}{\mathrm{bo}} T[U] \zeta_{x}+O\left(\mu^{3}\right) .
\end{aligned}\right.
$$

Now, denote by $\mathfrak{I}+2 \mu^{2} \mathfrak{T}[h]=h+\mu \mathcal{T}[h]+\mu^{2} \mathfrak{T}[h]$ [i.e., set $\pm \mu^{2} \mathfrak{T}[h]\left(\partial_{t} v+\varepsilon v v_{x}\right)$ in $\left.(27)_{2}\right]$ and note that from $(26)_{2}$, [i.e., applying a BBM trick (Benjamin-Bona-Mahony ${ }^{23}$ )], we get the following approximated equation 


$$
\partial_{t} v+\varepsilon v \partial_{x} v=-\partial_{x} \zeta+O(\mu) .
$$

Furthermore, substitute (28) in the acquired new term $2 \mu^{2} \mathfrak{T}[h]\left(\partial_{t} v+\varepsilon v v_{x}\right)$ to get $-2 \mu^{2} \mathfrak{T}[h] \zeta_{x}$, then after some computations [needed especially for a suitable specification of a new operator $\mathfrak{J}_{\text {bo }}$ introduced in (39)] we obtain the one-dimensional extended Green-Naghdi system with surface tension rewritten into

$$
\left\{\begin{array}{l}
\partial_{t} \zeta+\partial_{x}(h v)=0, \\
\mathfrak{J}\left(\partial_{t} v+\varepsilon v v_{x}\right)+h \partial_{x} \zeta-\frac{1}{\mathrm{bo}} \mu h \zeta_{x x x}+\frac{2}{45} \mu^{2} h \partial_{x}^{2}\left(h^{4} \zeta_{x x x}\right)+\mu^{2}\left(I_{1}[h] \zeta_{x}+I_{2}[h] \zeta_{x}\right) \\
\quad-\varepsilon^{2} \mu^{2} \frac{1}{\mathrm{bo}} T[U] \zeta_{x}+\varepsilon \mu \mathcal{Q}_{1}[U] v_{x}+\varepsilon \mu^{2} \mathcal{Q}_{2}[U] v_{x}+\varepsilon \mu^{2} \mathcal{Q}_{3}[U] v_{x}=O\left(\mu^{3}\right)
\end{array}\right.
$$

where $U=(\zeta, v)^{T}, h(t, x)=1+\varepsilon \zeta(t, x)$, and

$$
\begin{gathered}
\mathcal{T}[h] w=-\frac{1}{3} \partial_{x}\left(h^{3} \partial_{x} w\right), \quad \mathfrak{T}[h] w=-\frac{1}{45} \partial_{x}^{2}\left(h^{5} \partial_{x}^{2} w\right), \quad T[U] f=-\frac{1}{2} h \partial_{x}^{2}\left(\zeta_{x}^{2} f\right), \\
\mathcal{Q}_{1}[U] f=\frac{2}{3} \partial_{x}\left(h^{3} v_{x} f\right), \quad \mathcal{Q}_{2}[U] f=\frac{8}{45} \partial_{x}^{2}\left(h^{5} v_{x x} f\right), \quad \mathcal{Q}_{3}[U] f=\frac{1}{15} \partial_{x}\left(h^{5} v_{x x} \partial_{x} f\right), \\
I_{1}[h] f=\frac{4}{45} h_{x} \partial_{x}\left(h^{4} \partial_{x}^{2} f\right) \quad \text { and } \quad I_{2}[h] f=\frac{2}{45} h^{4} h_{x x} \partial_{x}^{2} f
\end{gathered}
$$

Remark 1. The interest of formulation (27) is that all the fifth order derivatives of $v$ are factorized in $\left(h+\mu \mathcal{T}[h]+\mu^{2} \mathfrak{T}[h]\right)\left(\partial_{t} v+\varepsilon v v_{x}\right)$ which is of notable help. However, the benefit of formulation (29) is in replacing $h+\mu \mathcal{T}[h]+\mu^{2} \mathfrak{T}[h]$ by a new operator $\mathfrak{J}$ so that the coercivity condition of the bilinear form is satisfied when applying a Lax-Millgram theorem for the proof of the invertibility of $\mathfrak{I}$ (see Lemma 1).

Remark 2. The reason for considering the effect of surface tension (see Ref. 1 for a brief physical relevance) is due to the betterment needed on the natural associated energy norm $\left.|\cdot|\right|_{\text {s }}[$ i.e., when no suface tension $\left(\mathrm{bo}^{-1}=0\right)$ ] defined by

$$
|(\zeta, v)|_{Y^{s}}^{2}=|\zeta|_{H^{s}}^{2}+\mu^{2}\left|\zeta_{x x}\right|_{H^{s}}^{2}+|v|_{H^{s}}^{2}+\mu\left|v_{x}\right|_{H^{s}}^{2}+\mu^{2}\left|v_{x x}\right|_{H^{s}}^{2} .
$$

Because the norm $|\cdot|_{Y s}$ is not adequate for the proof of the energy estimate (see for instance the control of the term $A_{2}+A_{3}$ ), the addition of the quantities in (25) permits the definition of a new energy norm,

$$
|(\zeta, v)|_{X^{s}}^{2}=|\zeta|_{H^{s}}^{2}+\frac{\mu}{\mathrm{bo}}\left|\zeta_{x}\right|_{H^{s}}^{2}+\mu^{2}\left|\zeta_{x x}\right|_{H^{s}}^{2}+|v|_{H^{s}}^{2}+\mu\left|v_{x}\right|_{H^{s}}^{2}+\mu^{2}\left|v_{x x}\right|_{H^{s}}^{2}
$$

The second term in $|\cdot|_{X^{s}}$ is absent from the natural energy $\left.|\cdot|\right|_{Y^{s}}$, and this will be a fundamental term for allowing the control of several inconvenient terms as we are going to figure it out in Sec. III B. Moreover, the space $\left\{\zeta \in H^{s}(\mathbb{R}) ;|\zeta|_{H^{s}}^{2}+\mu^{2}\left|\zeta_{x x}\right|_{H^{s}}^{2}<\infty\right\}$ is not equivalent to the Sobolev space $H^{s+2}(\mathbb{R})$, which required in defining the energy space $X^{s}$ of our problem (see Definition 1).

\section{A. Preliminary results}

Under the nonzero depth-condition,

$$
\exists \quad h_{\min }>0, \quad \inf _{x \in \mathbb{R}} h \geq h_{\min } \text { where } h(t, x)=1+\varepsilon \zeta(t, x),
$$

which is a fundamental condition for the extended Green-Naghdi system to be physically legitimate, and it says that the water bottom is constantly limited from underneath by a nonnegative constant.

We introduce the operator

$$
\mathfrak{I}=h+\mu \mathcal{T}[h]-\mu^{2} \mathfrak{T}[h]=h-\frac{1}{3} \mu \partial_{x}\left(h^{3} \partial_{x} \cdot\right)+\frac{1}{45} \mu^{2} \partial_{x}^{2}\left(h^{5} \partial_{x}^{2} \cdot\right),
$$


which plays an important role in the energy estimate and the local well-posedness of the extended Green-Naghdi system. We shall give an essential invertibility result on $\mathfrak{J}$ and specify some properties on its inverse $\mathfrak{J}^{-1}$, which are explained in the following lemmas.

Lemma 1. Assume that $\zeta(t, \cdot) \in L^{\infty}(\mathbb{R})$ is a differentiable scalar function under condition (33). Then, the operator

$$
\mathfrak{J}: H_{\mu}^{2}(\mathbb{R}) \longrightarrow L^{2}(\mathbb{R})
$$

is well defined, one-to-one and onto.

Proof. The proof of the invertibility of $\mathfrak{I}$ is a direct application on the Lax-Millgram theorem. We introduce first the space $H_{\mu}^{2}(\mathbb{R})$ endowed with the norm $|\cdot|_{\mu}$ defined as

$$
H_{\mu}^{2}(\mathbb{R})=\left\{v \in H^{2}(\mathbb{R}) ;|v|_{\mu}^{2}=|v|_{2}^{2}+\mu\left|v_{x}\right|_{2}^{2}+\mu^{2}\left|v_{x x}\right|_{2}^{2}<\infty\right\},
$$

where $|\cdot|_{\mu}$ is equivalent to $|\cdot|_{H^{2}}$ but not uniformly with respect to $\mu$.

Let $f \in L^{2}(\mathbb{R})$. Consider the weak problem

$$
\left\{\begin{array}{l}
\text { Find } \quad v \in H_{\mu}^{2}(\mathbb{R}) \quad \text { such that } \\
a(v, u)=L(u) \quad \forall u \in H_{\mu}^{2}(\mathbb{R}),
\end{array}\right.
$$

with

$$
a(v, u)=(\mathfrak{J} v, u)=(h v, u)+\frac{1}{3} \mu\left(h^{3} v_{x}, u_{x}\right)+\frac{1}{45} \mu^{2}\left(h^{5} v_{x x}, u_{x x}\right), \quad L(u)=(f, u) .
$$

It is easy to see that $a$ and $L$ are continuous on $H_{\mu}^{2}(\mathbb{R}) \times H_{\mu}^{2}(\mathbb{R})$ and $H_{\mu}^{2}(\mathbb{R})$, respectively. In addition, $a$ is coercive on $H_{\mu}^{2}(\mathbb{R}) \times H_{\mu}^{2}(\mathbb{R})$ using (33) with

$$
a(v, v) \geq \min \left(h_{\min }, \frac{1}{3} h_{\min }^{3}, \frac{1}{45} h_{\min }^{5}\right)|v|_{\mu}^{2} .
$$

Therefore by Lax-Millgram theorem, for every $f \in L^{2}(\mathbb{R})$, there exists a unique $v \in H_{\mu}^{2}(\mathbb{R})$ such that for all $u \in H_{\mu}^{2}(\mathbb{R})$, we have

$$
a(v, u)=(\mathfrak{J} v, u)=L(u)=(f, u) .
$$

Hence the result.

The following lemma gives functional properties to the operator $\mathfrak{J}^{-1}$.

Lemma 2. Let $t_{0}>\frac{1}{2}$ and $\zeta \in H^{t_{0}+1}(\mathbb{R})$ be such that (33) is satisfied. Then, we have the following:

(i) $\forall 0 \leq s \leq t_{0}+1$,

$$
\left|\mathfrak{J}^{-1} f\right|_{H^{s}}+\sqrt{\mu}\left|\partial_{x} \mathfrak{J}^{-1} f\right|_{H^{s}}+\mu\left|\partial_{x}^{2} \mathfrak{J}^{-1} f\right|_{H^{s}} \leq C\left(\frac{1}{h_{\min }},|h-1|_{H^{t_{0}+1}}\right)|f|_{H^{s}} .
$$

(ii) $\forall 0 \leq s \leq t_{0}+1$,

$$
\sqrt{\mu}\left|\mathfrak{J}^{-1} \partial_{x} f\right|_{H^{s}}+\mu\left|\partial_{x} \mathfrak{J}^{-1} \partial_{x} f\right|_{H^{s}}+\mu \sqrt{\mu}\left|\partial_{x}^{2} \mathfrak{J}^{-1} \partial_{x} f\right|_{H^{s}} \leq C\left(\frac{1}{h_{\min }},|h-1|_{H^{t_{0}+1}}\right)|f|_{H^{s}},
$$

and

$$
\mu\left|\mathfrak{J}^{-1} \partial_{x}^{2} f\right|_{H^{s}}+\mu \sqrt{\mu}\left|\partial_{x} \mathfrak{J}^{-1} \partial_{x}^{2} f\right|_{H^{s}}+\mu^{2}\left|\partial_{x}^{2} \mathfrak{J}^{-1} \partial_{x}^{2} f\right|_{H^{s}} \leq C\left(\frac{1}{h_{\min }},|h-1|_{H^{t^{+1}}}\right)|f|_{H^{s}} .
$$

(iii) $\forall s \geq t_{0}+1$,

$$
\left\|\mathfrak{J}^{-1}\right\|_{H^{s}(\mathbb{R}) \rightarrow H^{s}(\mathbb{R})}+\sqrt{\mu}\left\|\mathfrak{J}^{-1} \partial_{x}\right\|_{H^{s}(\mathbb{R}) \rightarrow H^{s}(\mathbb{R})}+\mu\left\|\mathfrak{J}^{-1} \partial_{x}^{2}\right\|_{H^{s}(\mathbb{R}) \rightarrow H^{s}(\mathbb{R})} \leq C_{s},
$$

with

$$
\mu\left\|\partial_{x} \mathfrak{J}^{-1} \partial_{x}\right\|_{H^{s}(\mathbb{R}) \rightarrow H^{s}(\mathbb{R})}+\mu \sqrt{\mu}\left\|\partial_{x}^{2} \mathfrak{J}^{-1} \partial_{x}\right\|_{H^{s}(\mathbb{R}) \rightarrow H^{s}(\mathbb{R})} \leq C_{s},
$$


and

$$
\mu \sqrt{\mu}\left\|\partial_{x} \mathfrak{J}^{-1} \partial_{x}^{2}\right\|_{H^{s}(\mathbb{R}) \rightarrow H^{s}(\mathbb{R})}+\mu^{2}\left\|\partial_{x}^{2} \mathfrak{J}^{-1} \partial_{x}^{2} f\right\|_{H^{s}(\mathbb{R}) \rightarrow H^{s}(\mathbb{R})} \leq C_{s},
$$

where $C_{s}$ is a constant depending on $1 / h_{\min }$ and $|h-1|_{H^{s}}$ and independent of $(\varepsilon, \mu) \in(0,1)^{2}$.

Proof. The proof is adapted as in Ref. 2 for $1 D$ Green-Naghdi equations ( $\mu^{2}$ order with $\beta \neq 0$ ). Assume that $f \in H^{s}(\mathbb{R})$ and $u=\mathfrak{J}^{-1} f$, then $\mathfrak{J} u=f$. Apply $\Lambda^{s}$ to both sides, then one can deduce the following:

$$
a\left(\Lambda^{s} u, \Lambda^{s} u\right)=\left(\widetilde{f}, \Lambda^{s} u\right)+\sqrt{\mu}\left(\partial_{x} \widetilde{g}, \Lambda^{s} u\right)+\mu\left(\partial_{x}^{2} \widetilde{p}, \Lambda^{s} u\right),
$$

where $\widetilde{f}, \widetilde{g}$, and $\widetilde{p}$ are written as follows:

$$
\widetilde{f}=\Lambda^{s} f-\left[\Lambda^{s}, h\right] u, \quad \widetilde{g}=\frac{1}{3} \sqrt{\mu}\left[\Lambda^{s}, h^{3}\right] u_{x}, \quad \widetilde{p}=-\frac{1}{45} \mu\left[\Lambda^{s}, h^{5}\right] u_{x x} .
$$

Integrating by parts and using (36), we get

$$
\min \left(h_{\text {min }}, \frac{1}{3} h_{\text {min }}^{3}, \frac{1}{45} h_{\text {min }}^{5}\right)\left|\Lambda^{s} u\right|_{\mu} \leq|\widetilde{f}|_{2}+|\widetilde{g}|_{2}+|\widetilde{p}|_{2} .
$$

Now, using the Kato-Pance commutator estimate (see Lemma 4.6 of Ref. 14), we have

$$
\left|\left[\Lambda^{s}, f\right] u\right|_{2} \lesssim|\nabla f|_{H^{t_{0}}}|u|_{H^{s-1}} .
$$

One can deduce

$$
|\widetilde{f}|_{2}+|\widetilde{g}|_{2}+|\widetilde{p}|_{2} \leq|f|_{H^{s}}+C\left(|h-1|_{H^{t_{0}+1}}\right)\left|\Lambda^{s-1} u\right|_{\mu} .
$$

Hence, the inequality (i) holds after continuous induction on $s$. For the proof of (ii), one has to replace $u=\sqrt{\mu} \mathfrak{J}^{-1} \partial_{x} f$ and $u=\mu \mathfrak{J}^{-1} \partial_{x}^{2} f$ for a second time. The general strategy is the same as in (i) noticing that $\Lambda^{s}$ commutes with $\partial_{x}, \partial_{x}^{2}$. The only difference is in the expression of $\widetilde{f}, \widetilde{g}$, and $\widetilde{p}$. In fact, when $u=\sqrt{\mu} \mathfrak{J}^{-1} \partial_{x} f$, we have the following:

$$
\widetilde{f}=-\left[\Lambda^{s}, h\right] u, \quad \widetilde{g}=\Lambda^{s} f+\frac{1}{3} \sqrt{\mu}\left[\Lambda^{s}, h^{3}\right] u_{x} \quad \text { and } \quad \widetilde{p}=-\frac{1}{45} \mu\left[\Lambda^{s}, h^{5}\right] u_{x x},
$$

and when $u=\mu \mathfrak{J}^{-1} \partial_{x}^{2} f$, we have the following:

$$
\widetilde{f}=-\left[\Lambda^{s}, h\right] u, \quad \widetilde{g}=\frac{1}{3} \sqrt{\mu}\left[\Lambda^{s}, h^{3}\right] u_{x} \quad \text { and } \quad \widetilde{p}=\Lambda^{s} f-\frac{1}{45} \mu\left[\Lambda^{s}, h^{5}\right] u_{x x} .
$$

In (iii), since $s \geq t_{0}+1$, then the Kato-Pance comutator estimate (37) is given by $\forall f \in H^{s}(\mathbb{R})$, $u \in H^{s-1}(\mathbb{R})$

$$
\left|\left[\Lambda^{s}, f\right] u\right|_{2} \lesssim|\nabla f|_{H^{s-1}}|u|_{H^{s-1}},
$$

and one has $H^{s}(\mathbb{R}) \hookrightarrow H^{t_{0}+1}(\mathbb{R})$. Now, proceeding in the same way as in (i), we get the same result with the constant $C_{s}$ depending on $1 / h_{\min }$ and $|h-1|_{H^{s}}$.

\section{B. Linear analysis}

In order to rewrite the extended Green-Naghdi system (for flat bottoms) with surface tension in a condensed form, we introduce a new operator $\mathfrak{J}_{\text {bo }}$,

$$
U=(\zeta, v)^{T}, \quad \mathfrak{J}_{\mathrm{bo}}=1-\frac{\mu}{\mathrm{bo}} \partial_{x}^{2}(\cdot)+\frac{2}{45} \mu^{2} \partial_{x}^{2}\left(h^{4} \partial_{x}^{2} \cdot\right), \quad h(t, x)=1+\varepsilon \zeta(t, x) .
$$

The first equation in (29) can be written as

$$
\partial_{t} \zeta+\varepsilon v \partial_{x} \zeta+h \partial_{x} v=0
$$

For the second equation in (29), applying $\mathfrak{I}^{-1}$ to both sides, we get

$$
\begin{aligned}
\partial_{t} v+\varepsilon v v_{x}+\mathfrak{J}^{-1}\left(h \mathfrak{J}_{\mathrm{bo}} \zeta_{x}\right) & +\mu^{2} \mathfrak{J}^{-1}\left(I_{1}[h] \zeta_{x}+I_{2}[h] \zeta_{x}\right)-\varepsilon^{2} \mu^{2} \frac{1}{\mathrm{bo}} \mathfrak{J}^{-1}\left(T[U] \zeta_{x}\right) \\
& +\varepsilon \mu \mathfrak{J}^{-1}\left(\mathcal{Q}_{1}[U] v_{x}\right)+\varepsilon \mu^{2} \mathfrak{J}^{-1}\left(\mathcal{Q}_{2}[U] v_{x}\right)+\varepsilon \mu^{2} \mathfrak{J}^{-1}\left(\mathcal{Q}_{3}[U] v_{x}\right)=O\left(\mu^{3}\right) .
\end{aligned}
$$


Hence the extended Green-Naghdi system $(\beta=0)$ with surface tension can be written in the form

$$
\partial_{t} U+A[U] \partial_{x} U=0
$$

where

$$
A[U]=\left(\begin{array}{c}
\varepsilon v \\
\mathfrak{J}^{-1}\left(h \mathfrak{J}_{\mathrm{bo}} \cdot\right)+\mu^{2} I-\varepsilon^{2} \mu^{2} \frac{1}{\mathrm{bo}} \mathfrak{J}^{-1}(T[U] \cdot) \varepsilon v+\varepsilon \mu \mathfrak{J}^{-1}\left(\mathcal{Q}_{1}[U] \cdot\right)+\varepsilon \mu^{2} Q
\end{array}\right),
$$

with

$$
I=\mathfrak{J}^{-1}\left(I_{1}[h] \cdot\right)+\mathfrak{J}^{-1}\left(I_{2}[h] \cdot\right) \quad \text { and } \quad Q=\mathfrak{J}^{-1}\left(\mathcal{Q}_{2}[U] \cdot\right)+\mathfrak{J}^{-1}\left(\mathcal{Q}_{3}[U] \cdot\right) .
$$

Now, consider the linearized system around some reference state $\underline{U}=(\underline{\zeta}, \underline{v})^{T}$,

$$
\left\{\begin{array}{l}
\partial_{t} U+A[\underline{U}] \partial_{x} U=0, \\
U_{l_{t=0}}=U_{0} .
\end{array}\right.
$$

The proof of the energy estimate which permits the convergence of an iterative scheme to construct a solution to the extended Green Naghdi system (29) with surface tension for the initial value problem (43) requires one to define the $X^{s}$ spaces, which are the energy spaces for this problem.

Definition 1. For all $s \geq 0$ and $T>0$, we denote by $X^{s}$ the vector space $H^{s+2}(\mathbb{R}) \times H^{s+2}(\mathbb{R})$ endowed with the norm

$$
|U|_{X^{s}}^{2}:=|\zeta|_{H^{s}}^{2}+\frac{\mu}{\mathrm{bo}}\left|\zeta_{x}\right|_{H^{s}}^{2}+\mu^{2}\left|\zeta_{x x}\right|_{H^{s}}^{2}+|v|_{H^{s}}^{2}+\mu\left|v_{x}\right|_{H^{s}}^{2}+\mu^{2}\left|v_{x x}\right|_{H^{s}}^{2}
$$

while $X_{T}^{s}$ stands for $C\left(\left[0, \frac{T}{\varepsilon}\right] ; X^{s}\right)$ endowed with its canonical norm.

First, recall that a pseudo-symmetrizer for $A[\underline{U}]$ is given by

$$
S=\left(\begin{array}{cc}
\underline{\mathfrak{J}}_{\mathrm{bo}} & 0 \\
0 & \underline{\mathfrak{J}}
\end{array}\right),
$$

with $\underline{h}=1+\varepsilon \underline{\zeta}, \underline{\mathfrak{I}}=\underline{h}+\mu \underline{\mathcal{T}}[\underline{h}]-\mu^{2} \underline{\mathfrak{T}}[\underline{h}]$, and $\underline{\mathfrak{J}}_{\mathrm{bo}}=1-\frac{\mu}{\mathrm{bo}} \partial_{x}^{2}(\cdot)+\frac{2}{45} \mu^{2} \partial_{x}^{2}\left(\underline{h}^{4} \partial_{x}^{2} \cdot\right)$. A natural energy for the initial value problem (43) is suggested to be

$$
E^{s}(U)^{2}=\left(\Lambda^{s} U, S \Lambda^{s} U\right)
$$

The connection between $E^{s}(U)$ and the $X^{s}$-norm is examined using the lemma below.

Lemma 3. Let $s \geq 0$ and $\zeta \in L^{\infty}(\mathbb{R})$. Under the depth-condition

$$
\exists \quad h_{\min }>0, \quad \inf _{x \in \mathbb{R}} h \geq h_{\text {min }}, \quad h(t, x)=1+\varepsilon \zeta(t, x),
$$

$E^{s}(U)$ is uniformly equivalent to the $|\cdot|_{X^{s}-n o r m}$ with respect to $(\mu, \varepsilon) \in(0,1)^{2}$,

$$
E^{s}(U) \leq C\left(\frac{1}{h_{\min }},|\underline{h}|_{\infty}\right)|U|_{X^{s}} \quad \text { and } \quad|U|_{X^{s}} \leq C\left(h_{\min }, \frac{1}{h_{\min }}\right) E^{s}(U) .
$$

Proof. First note that, $E^{s}(U)^{2}=\left(\Lambda^{s} U, S \Lambda^{s} U\right)$ with $S \Lambda^{s} U=\left(\underline{\mathfrak{J}}_{\mathrm{bo}} \Lambda^{s} \zeta, \underline{\mathfrak{I}} \Lambda^{s} v\right)$. Then we get

$$
E^{s}(U)^{2}=\left(\Lambda^{s} \zeta, \underline{\mathfrak{I}}_{\mathrm{bo}} \Lambda^{s} \zeta\right)+\left(\Lambda^{s} v, \underline{\mathfrak{I}} \Lambda^{s} v\right)
$$

Using the expression of $\underline{\mathfrak{I}}, \underline{\mathfrak{J}}_{\mathrm{bo}}$ integrating by parts, we get

$$
\begin{aligned}
E^{s}(U)^{2}= & \left(\Lambda^{s} \zeta, \Lambda^{s} \zeta\right)+\frac{\mu}{\mathrm{bo}}\left(\Lambda^{s} \zeta_{x}, \Lambda^{s} \zeta_{x}\right)+\frac{2}{45} \mu^{2}\left(\underline{h}^{4} \Lambda^{s} \zeta_{x x}, \Lambda^{s} \zeta_{x x}\right)+\left(\Lambda^{s} v, \underline{h} \Lambda^{s} v\right) \\
& +\frac{\mu}{3}\left(\Lambda^{s} v_{x}, \underline{h}^{3} \Lambda^{s} v_{x}\right)+\frac{1}{45} \mu^{2}\left(\Lambda^{s} v_{x x}, \underline{h}^{5} \Lambda^{s} v_{x x}\right),
\end{aligned}
$$


but $\zeta \in L^{\infty}(\mathbb{R})$, and using the fact that the water depth is always bounded from below by a nonnegative constant (33), with the help of the Cauchy-Schwartz inequality, we get the following two inequalities:

$E^{s}(U)^{2} \leq \max \left\{1,|\underline{h}|_{\infty}, \frac{\left|\underline{h}^{3}\right|_{\infty}}{3}, 2 \frac{\left|\underline{h}^{4}\right|_{\infty}}{45}, \frac{\left|\underline{h}^{5}\right|_{\infty}}{45}\right\}|U|_{X^{s}}^{2}, E^{s}(U)^{2} \geq \min \left\{1, h_{\min }, \frac{h_{\min }^{3}}{3}, 2 \frac{h_{\min }^{4}}{45}, \frac{h_{\min }^{5}}{45}\right\}|U|_{X^{s}}^{2}$.

Finally, there exists $m, M$ greater than zero such that $\max \left\{1,|\underline{h}|_{\infty}, \frac{\left|\underline{h}^{3}\right|_{\infty}}{3}, 2 \frac{\left|h^{4}\right|_{\infty}}{45}, \frac{\left|h^{5}\right|_{\infty}}{45}\right\} \leq M$ and $\min \left\{1, h_{\min }, \frac{h_{\min }^{3}}{3}, 2 \frac{h_{\min }^{4}}{45}, \frac{h_{\min }^{5}}{45}\right\} \geq m$. Hence the lemma is confirmed.

The proof of the energy estimate is given in the following proposition.

Proposition 1. Let $t_{0}>\frac{1}{2}$ and $s \geq t_{0}+1$. Let also $\underline{U}=(\underline{\zeta}, \underline{v})^{T} \in X_{T}^{s}$ be such that $\partial_{t} \underline{U} \in X_{T}^{s-1}$ satisfying condition (33) on $\left[0, \frac{T}{\varepsilon}\right]$. Then, for all $U_{0} \in X^{s}$, there exists a unique solution $U=(\zeta, v)^{T} \in$ $X_{T}^{s}$ to (43) and for all $0 \leq t \leq \frac{T}{\varepsilon}$ satisfying

$$
E^{s}(U(t)) \leq\left(e^{\varepsilon \lambda_{T} t}\right)^{1 / 2} E^{s}\left(U_{0}\right),
$$

for some $\lambda_{T}=\lambda_{T}\left(\sup _{0 \leq t \leq T / \varepsilon} E^{S}(\underline{U}(t)), \sup _{0 \leq t \leq T / \varepsilon}\left|\partial_{t} \underline{h}(t)\right|_{L^{\infty}}\right)$.

Proof. For any $\lambda \in \mathbb{R}$, we compute

$$
e^{\varepsilon \lambda t} \partial_{t}\left(e^{-\varepsilon \lambda t} E^{s}(U)^{2}\right)=-\varepsilon \lambda E^{s}(U)^{2}+\partial_{t}\left(E^{s}(U)^{2}\right)
$$

with

$$
\partial_{t}\left(E^{s}(U)^{2}\right)=\left(\Lambda^{s} \zeta_{t}, \underline{\mathfrak{I}}_{\mathrm{bo}} \Lambda^{s} \zeta\right)+\left(\Lambda^{s} \zeta, \partial_{t}\left(\underline{\mathfrak{I}}_{\mathrm{bo}} \Lambda^{s} \zeta\right)\right)+\left(\Lambda^{s} v, \underline{\mathfrak{I}} \Lambda^{s} v_{t}\right)+\left(\Lambda^{s} v, \partial_{t}\left(\underline{\mathfrak{I}} \Lambda^{s} v\right)\right) .
$$

Using the fact that $\mathfrak{I}$ and $\underline{\mathfrak{I}}_{\text {bo }}$ are symmetric, in addition to the identities

$$
\partial_{t}\left(\underline{\mathfrak{I}} \Lambda^{s} v\right)=\left[\partial_{t}, \underline{\mathfrak{I}}\right] \Lambda^{s} v+\underline{\mathfrak{I}} \Lambda^{s} v_{t} \quad \partial_{t}\left(\underline{\mathfrak{I}}_{\mathrm{bo}} \Lambda^{s} \zeta\right)=\left[\partial_{t}, \underline{\mathfrak{I}}_{\mathrm{bo}}\right] \Lambda^{s} \zeta+\underline{\mathfrak{I}}_{\mathrm{bo}} \Lambda^{s} \zeta_{t},
$$

one gets after using (43) the following:

$$
\begin{aligned}
\partial_{t}\left(E^{s}(U)^{2}\right)= & -2\left(S \Lambda^{s} U,\left[\Lambda^{s}, A[\underline{U}]\right] \partial_{x} U\right)-2\left(S \Lambda^{s} U, A[\underline{U}] \Lambda^{s} \partial_{x} U\right)+\left(\Lambda^{s} \zeta,\left[\partial_{t}, \underline{\mathfrak{J}}_{\mathrm{bo}}\right] \Lambda^{s} \zeta\right) \\
& +\left(\Lambda^{s} v,\left[\partial_{t}, \underline{\mathfrak{J}}\right] \Lambda^{s} v\right) .
\end{aligned}
$$

Therefore, we obtain

$$
\begin{aligned}
\frac{1}{2} e^{\varepsilon \lambda t} \partial_{t}\left(e^{-\varepsilon \lambda t} E^{s}(U)^{2}\right)= & -\frac{\varepsilon \lambda}{2} E^{s}(U)^{2}-\left(S A[\underline{U}] \Lambda^{s} \partial_{x} U, \Lambda^{s} U\right)-\left(\left[\Lambda^{s}, A[\underline{U}]\right] \partial_{x} U, S \Lambda^{s} U\right) \\
& +\frac{1}{2}\left(\Lambda^{s} \zeta,\left[\partial_{t}, \underline{\mathfrak{J}}_{\mathrm{bo}}\right] \Lambda^{s} \zeta\right)+\frac{1}{2}\left(\Lambda^{s} v,\left[\partial_{t}, \underline{\mathfrak{I}}\right] \Lambda^{s} v\right) .
\end{aligned}
$$

- Estimation of $\left(S A[\underline{U}] \Lambda^{s} \partial_{x} U, \Lambda^{s} U\right)$. Noting that

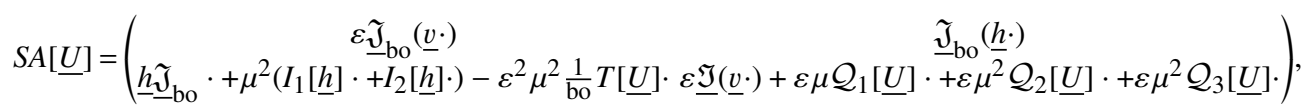

we get

$$
\begin{aligned}
\left(S A[\underline{U}] \Lambda^{s} \partial_{x} U, \Lambda^{s} U\right)= & \varepsilon\left(\underline{\mathfrak{J}}_{\mathrm{bo}}\left(\underline{v}^{s} \zeta_{x}\right), \Lambda^{s} \zeta\right)+\left(\underline{\mathfrak{J}}_{\mathrm{bo}}\left(\underline{h}^{s} v_{x}\right), \Lambda^{s} \zeta\right)+\left(\underline{h} \underline{\mathfrak{J}}_{\mathrm{bo}} \Lambda^{s} \zeta_{x}, \Lambda^{s} v\right)+\mu^{2}\left(I_{1}[\underline{h}] \Lambda^{s} \zeta_{x}, \Lambda^{s} v\right) \\
& +\mu^{2}\left(I_{2}[\underline{h}] \Lambda^{s} \zeta_{x}, \Lambda^{s} v\right)-\varepsilon^{2} \mu^{2} \frac{1}{\mathrm{bo}}\left(T[\underline{U}] \Lambda^{s} \zeta_{x}, \Lambda^{s} v\right)+\varepsilon\left(\underline{\mathfrak{J}}\left(\underline{v} \Lambda^{s} v_{x}\right), \Lambda^{s} v\right) \\
& +\varepsilon \mu\left(\mathcal{Q}_{1}[\underline{U}] \Lambda^{s} v_{x}, \Lambda^{s} v\right)+\varepsilon \mu^{2}\left(\mathcal{Q}_{2}[\underline{U}] \Lambda^{s} v_{x}, \Lambda^{s} v\right)+\varepsilon \mu^{2}\left(\underline{\mathcal{Q}_{3}}[\underline{U}] \Lambda^{s} v_{x}, \Lambda^{s} v\right) \\
= & A_{1}+A_{2}+\cdots+A_{10} .
\end{aligned}
$$

To control $A_{1}$, we denote here and throughout the rest of the proof the notation $f_{x}^{m}=\left(f^{m}\right)_{x}$, and we write using integration by parts,

$$
A_{1}=\varepsilon\left(\underline{h v} \Lambda^{s} \zeta_{x}, \Lambda^{s} \zeta\right)+\varepsilon \frac{\mu}{\text { bo }}\left(\partial_{x}\left(\underline{v} \Lambda^{s} \zeta_{x}\right), \Lambda^{s} \zeta_{x}\right)+\frac{2}{45} \varepsilon \mu^{2}\left(\underline{h}^{4} \partial_{x}^{2}\left(\underline{v} \Lambda^{s} \zeta_{x}\right), \Lambda^{s} \zeta_{x x}\right)=A_{11}+A_{12}+A_{13} .
$$


We have

$$
\left|A_{11}\right|=\left|\frac{\varepsilon}{2}\left(\Lambda^{s} \zeta, \underline{h}_{x} \underline{v} \Lambda^{s} \zeta\right)+\frac{\varepsilon}{2}\left(\Lambda^{s} \zeta, \underline{h v}_{x} \Lambda^{s} \zeta\right)\right| \leq \varepsilon C\left(|\underline{\zeta}|_{W^{1, \infty}},|\underline{v}|_{W^{1, \infty}}\right) E^{s}(U)^{2} .
$$

Integrating by parts, $A_{12}$ yields the expression

$$
\left|A_{12}\right|=\varepsilon \frac{\mu}{\mathrm{bo}}\left|\left(\underline{v}_{x} \Lambda^{s} \zeta_{x}, \Lambda^{s} \zeta_{x}\right)-\frac{1}{2}\left(\Lambda^{s} \zeta_{x}, \underline{v}_{x} \Lambda^{s} \zeta_{x}\right)\right| \leq \varepsilon C\left(\left|\underline{v}_{x}\right|_{\infty}\right) E^{s}(U)^{2} .
$$

Now using the fact that

$$
\partial_{x}^{2}(M N)=N \partial_{x}^{2} M+2 M_{x} N_{x}+M \partial_{x}^{2} N,
$$

for any differentiable functions $M$ and $N$ and integrating by parts, we have

$$
\begin{aligned}
\left|A_{13}\right| & =\frac{2}{45} \varepsilon \mu^{2}\left|\left(\underline{h}^{4} \underline{v}_{x x} \Lambda^{s} \zeta_{x}, \Lambda^{s} \zeta_{x x}\right)+2\left(\underline{h}^{4} \underline{v}{ }_{x} \Lambda^{s} \zeta_{x x}, \Lambda^{s} \zeta_{x x}\right)-\frac{1}{2}\left(\underline{h}_{x}^{4} \underline{v} \Lambda^{s} \zeta_{x x}, \Lambda^{s} \zeta_{x x}\right)-\frac{1}{2}\left(\underline{h}^{4} \underline{v}_{x} \Lambda^{s} \zeta_{x x}, \Lambda^{s} \zeta_{x x}\right)\right| \\
& \leq \varepsilon C\left(|\zeta|_{W^{1, \infty}},|\underline{v}|_{W^{1, \infty}},\left.\left.\sqrt{\mu}\right|_{x x}\right|_{\infty}, \sqrt{\mathrm{bo}}\right) E^{s}(U)^{2} .
\end{aligned}
$$

Gathering the information provided above, we get

$$
\left|A_{1}\right| \leq \varepsilon C\left(|\underline{\zeta}|_{W^{1, \infty}},|\underline{v}|_{W^{1, \infty}},\left.\left.\sqrt{\mu}\right|_{\underline{v} x}\right|_{\infty}, \sqrt{\mathrm{bo}}\right) E^{s}(U)^{2} .
$$

To control $A_{2}+A_{3}$, we write using integration by parts and remarking that $\underline{\mathfrak{J}}_{\text {bo }}$ is symmetric,

$$
\begin{aligned}
A_{2}+A_{3}=\left(\Lambda^{s} v_{x}, \underline{h} \Lambda^{s} \zeta\right)+\left(\underline{h} \Lambda^{s} \zeta_{x}, \Lambda^{s} v\right)-\frac{\mu}{\mathrm{bo}}\left(\Lambda^{s} v_{x}, \underline{h} \Lambda^{s} \zeta_{x x}\right) \\
\quad-\frac{\mu}{\mathrm{bo}}\left(\Lambda^{s} \zeta_{x x x}, \underline{h} \Lambda^{s} v\right)+\frac{2}{45} \mu^{2}\left(\underline{h} \Lambda^{s} v_{x}, \partial_{x}^{2}\left(\underline{h}^{4} \Lambda^{s} \zeta_{x x}\right)\right)+\frac{2}{45} \mu^{2}\left(\underline{h} \Lambda^{s} v, \partial_{x}^{2}\left(\underline{h}^{4} \Lambda^{s} \zeta_{x x x}\right)\right) .
\end{aligned}
$$

Integrating by parts again with some computations and using (49), one gets

$$
\begin{aligned}
A_{2}+A_{3}= & -\left(\Lambda^{s} v, \underline{h}_{x} \Lambda^{s} \zeta\right)-\frac{\mu}{\mathrm{bo}}\left(\Lambda^{s} \zeta_{x}, \underline{h}_{x x} \Lambda^{s} v\right)-\frac{\mu}{\mathrm{bo}}\left(\Lambda^{s} \zeta_{x}, \underline{h}_{x} \Lambda^{s} v_{x}\right)+\frac{2}{45} \mu^{2}\left(\underline{h}_{x x} \Lambda^{s} v_{x}, \underline{h}^{4} \Lambda^{s} \zeta_{x x}\right) \\
& -\frac{2}{45} \mu^{2}\left(\Lambda^{s} \zeta_{x x}, \partial_{x}\left(\underline{h}^{4} \underline{h}_{x x} \Lambda^{s} v\right)\right)-\frac{4}{45} \mu^{2}\left(\Lambda^{s} \zeta_{x x}, \partial_{x}\left(\underline{h}^{4} \underline{h}_{x} \Lambda^{s} v_{x}\right)\right)-\frac{2}{45} \mu^{2}\left(\Lambda^{s} \zeta_{x x}, \underline{h}_{x}^{5} \Lambda^{s} v_{x x}\right) .
\end{aligned}
$$

Therefore, we have

$$
\left|A_{2}+A_{3}\right| \leq \varepsilon C\left(|\underline{\zeta}|_{W^{1, \infty}}, \sqrt{\frac{\mu}{\mathrm{bo}}}\left|\underline{\zeta}_{x}\right|_{H^{s}}\right) E^{s}(U)^{2} .
$$

To control $A_{4}$, an integration by parts yields

$$
\left|A_{4}\right|=\frac{4}{45}\left|\mu^{2}\left(\Lambda^{s} \zeta_{x x}, \partial_{x}\left(\underline{h}^{4} \underline{h}_{x x} \Lambda^{s} v\right)\right)+\mu^{2}\left(\Lambda^{s} \zeta_{x x}, \partial_{x}\left(\underline{h}^{4} \underline{h}_{x} \Lambda^{s} v_{x}\right)\right)\right| \leq \varepsilon C\left(\left|\underline{\zeta}_{W^{1, \infty}}, \sqrt{\frac{\mu}{\mathrm{bo}}}\right| \underline{\zeta}_{x}\left|H^{s}, \mu\right| \underline{\zeta}_{x x} \mid H^{s}\right) E^{s}(U)^{2} .
$$

To control $A_{5}$, an integration by parts yields

$$
\left|A_{5}\right|=\frac{2}{45}\left|-\mu^{2}\left(\Lambda^{s} \zeta_{x x}, \partial_{x}\left(\underline{h}^{4} \underline{h}_{x x} \Lambda^{s} v\right)\right)\right| \leq \varepsilon C\left(|\underline{\zeta}|_{W^{1, \infty}}, \sqrt{\frac{\mu}{\mathrm{bo}}}\left|\underline{\zeta}_{x}\right|_{H^{s}}, \mu\left|\underline{\zeta}_{x x}\right|_{H^{s}}\right) E^{s}(U)^{2} .
$$

To control $A_{6}$, we use integration by parts and (49) to get

$$
\begin{aligned}
\left|A_{6}\right|=\frac{1}{2 \mathrm{bo}} \varepsilon^{2} \mu^{2} \mid\left(\underline{\zeta}_{x}^{2} \Lambda^{s} \zeta_{x}, \underline{h} \Lambda^{s} v_{x x}\right)+2 & \left(\underline{\zeta}_{x}^{2} \Lambda^{s} \zeta_{x}, \underline{h}_{x} \Lambda^{s} v_{x}\right) \\
& +\left(\underline{\zeta}_{x}^{2} \Lambda^{s} \zeta_{x}, \underline{h}_{x x} \Lambda^{s} v\right) \mid \leq \varepsilon C\left(|\underline{\zeta}|_{W^{1, \infty}}, \sqrt{\frac{\mu}{\mathrm{bo}}}\left|\underline{\zeta}_{x}\right|_{H^{s}}\right) E^{s}(U)^{2} .
\end{aligned}
$$

To control $A_{7}$, we write using an integration by parts,

$$
A_{7}=\varepsilon\left(\underline{h v} \Lambda^{s} v_{x}, \Lambda^{s} v\right)+\frac{\varepsilon \mu}{3}\left(\underline{h}^{3} \partial_{x}\left(\underline{v} \Lambda^{s} v_{x}\right), \Lambda^{s} v_{x}\right)+\frac{\varepsilon \mu^{2}}{45}\left(\underline{h}^{5} \partial_{x}^{2}\left(\underline{v} \Lambda^{s} v_{x}\right), \Lambda^{s} v_{x x}\right)=A_{71}+A_{72}+A_{73},
$$

where

$$
\left|A_{71}\right|=\left|-\frac{\varepsilon}{2}\left(\underline{h}_{x} \underline{v} \Lambda^{s} v, \Lambda^{s} v\right)-\frac{\varepsilon}{2}\left(\underline{h}_{x} \Lambda^{s} v, \Lambda^{s} v\right)\right| \leq \varepsilon C\left(\left|\underline{\zeta}_{x}\right|_{\infty},\left|\underline{v}_{x}\right|_{\infty}\right) E^{s}(U)^{2}
$$

with

$$
\left|A_{72}\right|=\left|-\frac{\varepsilon \mu}{6}\left(\underline{h}_{x}^{3} \underline{v} \Lambda^{s} v_{x}, \Lambda^{s} v_{x}\right)-\frac{\varepsilon \mu}{6}\left(\underline{h}^{3} \underline{v} \Lambda^{s} v_{x}, \Lambda^{s} v_{x}\right)\right| \leq \varepsilon C\left(|\underline{\zeta}|_{W^{1, \infty}}\right) E^{s}(U)^{2}
$$


and

$$
\begin{aligned}
\left|A_{73}\right| & =\frac{\varepsilon \mu^{2}}{45}\left|\left(\underline{h}^{5} \underline{v}_{x x} \Lambda^{s} v_{x}, \Lambda^{s} v_{x x}\right)+\left(\underline{h}^{5} \underline{v}_{x} \Lambda^{s} v_{x x}, \Lambda^{s} v_{x x}\right)-\frac{1}{2}\left(\underline{h}_{x}^{5} \underline{v} \Lambda^{s} v_{x x}, \Lambda^{s} v_{x x}\right)-\frac{1}{2}\left(\underline{h}^{5} \underline{v}_{x} \Lambda^{s} v_{x x}, \Lambda^{s} v_{x x}\right)\right| \\
& \leq \varepsilon C\left(|\underline{\zeta}|_{W^{1, \infty}},\left.\sqrt{\mu} \underline{v}_{x x}\right|_{\infty}\right) E^{s}(U)^{2} .
\end{aligned}
$$

Hence, we get

$$
\left|A_{7}\right| \leq \varepsilon C\left(|\underline{\zeta}|_{W^{1, \infty}},\left.\underline{v}_{x}\right|_{\infty},\left.\sqrt{\mu} \underline{v}_{x x}\right|_{\infty}\right) E^{s}(U)^{2} .
$$

Finally, by a simple integration by parts, $A_{8}, A_{9}$, and $A_{10}$ are controlled by $C\left(|\underline{\zeta}|_{\infty},\left.\underline{v}_{x}\right|_{\infty},\left.\sqrt{\mu} \underline{v}_{x x}\right|_{\infty}\right)$ $E^{s}(U)^{2}$. Therefore, we have

$$
\left|\left(S A[\underline{U}] \Lambda^{s} \partial_{x} U, \Lambda^{s} U\right)\right| \leq \varepsilon C\left(|\zeta|_{W^{1, \infty}}, \sqrt{\frac{\mu}{\mathrm{bo}}}\left|\underline{\zeta}_{x}\right|_{H^{s}},\left.\left.\mu\right|_{\underline{\zeta}_{x x}}\right|_{H^{s}},|\underline{v}|_{W^{1, \infty}},\left.\left.\sqrt{\mu}\right|_{\underline{v} x}\right|_{\infty}, \sqrt{\mathrm{bo}}\right) E^{s}(U)^{2} .
$$

- Estimation of $\left(\left[\Lambda^{s}, A[\underline{U}]\right] \partial_{x} U, S \Lambda^{s} U\right)$. Note that

$$
\begin{aligned}
& \left(\left[\Lambda^{s}, A[\underline{U}]\right] \partial_{x} U, S \Lambda^{s} U\right)=\varepsilon\left(\left[\Lambda^{s}, \underline{v}\right] \zeta_{x}, \underline{\mathfrak{I}}_{\mathrm{bo}} \Lambda^{s} \zeta\right)+\varepsilon\left(\left[\Lambda^{s}, \underline{h}\right] v_{x}, \underline{\mathfrak{J}}_{\mathrm{bo}} \Lambda^{s} \zeta\right)+\left(\left[\Lambda^{s}, \underline{\mathfrak{I}}^{-1}\left(\underline{h}_{\mathrm{I}} \underline{\mathrm{J}}_{\mathrm{bo}} \cdot\right)\right] \zeta_{x}, \underline{\mathfrak{I}}^{s} v\right) \\
& +\mu^{2}\left(\left[\Lambda^{s}, \underline{\mathfrak{I}}^{-1}\left(I_{1}[\underline{h}] \cdot\right)\right] \zeta_{x}, \underline{\mathfrak{I}} \Lambda^{s} v\right)+\mu^{2}\left(\left[\Lambda^{s}, \underline{\mathfrak{I}}^{-1}\left(I_{2}[\underline{h}] \cdot\right)\right] \zeta_{x}, \underline{\mathfrak{I}} \Lambda^{s} v\right) \\
& -\varepsilon^{2} \mu^{2} \frac{1}{\mathrm{bo}}\left(\left[\Lambda^{s}, \underline{\mathfrak{I}}^{-1}(T[\underline{U}] \cdot)\right] \zeta_{x}, \underline{\mathfrak{I}} \Lambda^{s} v\right)+\varepsilon\left(\left[\Lambda^{s}, \underline{v}\right] v_{x}, \underline{\mathfrak{I}} \Lambda^{s} v\right)+\varepsilon \mu\left(\left[\Lambda^{s}, \underline{\mathfrak{I}}^{-1}\left(\mathcal{Q}_{1}[\underline{U}] \cdot\right)\right] v_{x}, \underline{\mathfrak{I}} \Lambda^{s} v\right) \\
& +\varepsilon \mu^{2}\left(\left[\Lambda^{s}, \underline{\mathfrak{I}}^{-1}\left(\mathcal{Q}_{2}[\underline{U}] \cdot\right)\right] v_{x}, \underline{\mathfrak{I}} \Lambda^{s} v\right)+\varepsilon \mu^{2}\left(\left[\Lambda^{s}, \underline{\mathfrak{I}}^{-1}\left(\mathcal{Q}_{3}[\underline{U}] \cdot\right)\right] v_{x}, \underline{\mathfrak{I}} \Lambda^{s} v\right) \\
& =B_{1}+B_{2}+\cdots+B_{10} \text {. }
\end{aligned}
$$

To control $B_{1}$, we use the expression of $\underline{\mathfrak{J}}_{\mathrm{bo}}$ to write

$$
B_{1}=\varepsilon\left(\left[\Lambda^{s}, \underline{v}\right] \zeta_{x}, \underline{h} \Lambda^{s} \zeta\right)-\frac{\varepsilon \mu}{\mathrm{bo}}\left(\left[\Lambda^{s}, \underline{v}\right] \zeta_{x}, \Lambda^{s} \zeta_{x x}\right)+\frac{2}{45} \varepsilon \mu^{2}\left(\partial_{x}^{2}\left[\Lambda^{s}, \underline{v}\right] \zeta_{x}, \underline{h}^{4} \Lambda^{s} \zeta_{x x}\right) .
$$

Then by using the fact that

$$
\partial_{x}^{2}\left[\Lambda^{s}, M\right] N=\left[\Lambda^{s}, M_{x x}\right] N+2\left[\Lambda^{s}, M_{x}\right] N_{x}+\left[\Lambda^{s}, M\right] N_{x x},
$$

one gets

$$
\begin{aligned}
B_{1}=\varepsilon\left(\left[\Lambda^{s}, \underline{v}\right] \zeta_{x}, \underline{h} \Lambda^{s} \zeta\right)-\frac{\varepsilon \mu}{\mathrm{bo}}\left(\left[\Lambda^{s}, \underline{v}\right] \zeta_{x}, \Lambda^{s} \zeta_{x x}\right) \\
+\frac{2}{45} \varepsilon \mu^{2}\left\{\left(\left[\Lambda^{s}, \underline{v}_{x x}\right] \zeta_{x}, \underline{h}^{4} \Lambda^{s} \zeta_{x x}\right)+2\left(\left[\Lambda^{s}, \underline{v}_{x}\right] \zeta_{x x}, \underline{h}^{4} \Lambda^{s} \zeta_{x x}\right)+\left(\left[\Lambda^{s}, \underline{v}\right] \zeta_{x x x}, \underline{h}^{4} \Lambda^{s} \zeta_{x x}\right)\right\} .
\end{aligned}
$$

Hence, using the commutator estimate (38), one obtains

$$
\left|B_{1}\right| \leq \varepsilon C\left(|\underline{\zeta}|_{\infty},|\underline{v}|_{H^{s}},\left.\mu \underline{v}_{x x}\right|_{H^{s}}, \sqrt{\mathrm{bo}}\right) E^{s}(U)^{2} .
$$

To control $B_{2}$, we use the expression of $\underline{\mathfrak{J}}_{\mathrm{bo}}$ and (50) to write

$$
\begin{aligned}
B_{2}=\varepsilon\left(\left[\Lambda^{s}, \underline{h}-1\right] v_{x}, \underline{h} \Lambda^{s} \zeta\right)-\frac{\varepsilon \mu}{\mathrm{bo}}\left(\left[\Lambda^{s}, \underline{h}-1\right] v_{x}, \Lambda^{s} \zeta_{x x}\right)+\frac{2}{45} \varepsilon \mu^{2}\left\{\left(\left[\Lambda^{s},(\underline{h}-1)_{x x}\right] v_{x}, \underline{h^{4}} \Lambda^{s} \zeta_{x x}\right)\right. \\
\left.+2\left(\left[\Lambda^{s},(\underline{h}-1)_{x}\right] v_{x x}, \underline{h}^{4} \Lambda^{s} \zeta_{x x}\right)+\left(\left[\Lambda^{s}, \underline{h}-1\right] v_{x x x}, \underline{h}^{4} \Lambda^{s} \zeta_{x x}\right)\right\} .
\end{aligned}
$$

Then, we obtain

$$
\left|B_{2}\right| \leq \varepsilon C\left(|\underline{h}-1|_{H^{s}}, \sqrt{\frac{\mu}{\mathrm{bo}}}\left|\underline{\zeta}_{x}\right|_{H^{s}}, \mu\left|\underline{\zeta}_{x x}\right|_{H^{s}}, \sqrt{\mathrm{bo}}\right) E^{s}(U)^{2} .
$$

To control $B_{3}+B_{4}+B_{5}$, remark that $\underline{\mathfrak{I}}$ is symmetric with

$$
\underline{\mathfrak{I}}\left[\Lambda^{s}, \underline{\mathfrak{I}}^{-1}\right] \underline{h}_{\mathfrak{J}_{\mathrm{bo}}} \zeta_{x}=\underline{\mathfrak{I}}\left[\Lambda^{s}, \underline{\mathfrak{I}}^{-1}\left(\underline{\mathfrak{J}}_{\mathrm{bo}} \cdot\right)\right] \zeta_{x}-\left[\Lambda^{s}, \underline{h} \underline{\mathfrak{J}}_{\mathrm{bo}}\right] \zeta_{x} .
$$

Moreover, since $\left[\Lambda^{s}, \underline{\mathfrak{I}}^{-1}\right]=-\underline{\mathfrak{I}}^{-1}\left[\Lambda^{s}, \underline{\mathfrak{I}}\right] \underline{\mathfrak{I}}^{-1}$, one gets

$$
\underline{\mathfrak{I}}\left[\Lambda^{s}, \underline{\mathfrak{I}}^{-1}\left(\underline{h} \underline{\mathfrak{J}}_{\mathrm{bo}} \cdot\right)\right] \zeta_{x}=-\left[\Lambda^{s}, \underline{\mathfrak{I}}\right] \underline{\mathfrak{I}}^{-1} \underline{\mathfrak{J}}_{\mathrm{bo}} \zeta_{x}+\left[\Lambda^{s}, \underline{\mathfrak{I}}_{\mathrm{bo}}\right] \zeta_{x} .
$$

By using the explicit expression of $\underline{\mathfrak{I}}$, integration by parts, and the fact that

$$
\left[\Lambda^{s}, \partial_{x}\left(M \partial_{x} \cdot\right)\right] N=\partial_{x}\left[\Lambda^{s}, M\right] N_{x}, \quad\left[\Lambda^{s}, \partial_{x}^{2}\left(M \partial_{x}^{2} \cdot\right)\right] N=\partial_{x}^{2}\left[\Lambda^{s}, M\right] N_{x x},
$$


one can write

$$
\begin{aligned}
B_{3}+B_{4}+B_{5} & =\left(\left[\Lambda^{s}, \underline{\mathfrak{I}}\right]^{\mathfrak{I}}{ }^{-1}\left(\underline{h} \underline{\mathfrak{J}}_{\mathrm{bo}} \zeta_{x}\right), \Lambda^{s} v\right)+\left(\left[\Lambda^{s}, \underline{h}_{\mathrm{J}}\right] \zeta_{x}, \Lambda^{s} v\right)+\mu^{2}\left(\left[\Lambda^{s}, \underline{\mathfrak{I}} \underline{\mathfrak{I}}^{-1}\left(I_{1}[\underline{h}] \zeta_{x}\right), \Lambda^{s} v\right)\right. \\
& +\mu^{2}\left(\left[\Lambda^{s}, I_{1}[\underline{h}]\right] \zeta_{x}, \Lambda^{s} v\right)+\mu^{2}\left(\left[\Lambda^{s}, \underline{\mathfrak{I}}\right] \underline{\mathfrak{I}}^{-1}\left(I_{2}\left[\underline{h} \zeta_{x}\right), \Lambda^{s} v\right)+\mu^{2}\left(\left[\Lambda^{s}, I_{2}[\underline{h}]\right] \zeta_{x}, \Lambda^{s} v\right) .\right.
\end{aligned}
$$

Now, using the expression of $\underline{\mathfrak{J}}_{\text {bo }}$ and (49), one has

$$
\frac{2}{45} \mu^{2} \underline{h} \partial_{x}^{2}\left(\underline{h}^{4} \zeta_{x x x}\right)=2 \underline{\mathfrak{J}} \zeta_{x}-2 \underline{h} \zeta_{x}+\frac{2 \mu}{3} \partial_{x}\left(\underline{h}^{3} \zeta_{x x}\right)-\mu^{2} I_{1}[\underline{h}] \zeta_{x}-\mu^{2} I_{2}[\underline{h}] \zeta_{x} .
$$

Then, we get

$\underline{\mathfrak{I}}^{-1}\left(\underline{h}_{\mathrm{J}} \zeta_{x}\right)=2 \zeta_{x}-\underline{\mathfrak{I}}^{-1}\left(\underline{h} \zeta_{x}\right)-\frac{\mu}{\mathrm{bo}} \underline{\mathfrak{I}}^{-1}\left(\underline{h} \zeta_{x x x}\right)+\frac{2 \mu}{3} \underline{\mathfrak{I}}^{-1} \partial_{x}\left(\underline{h}^{3} \zeta_{x x}\right)-\mu^{2} \underline{\mathfrak{I}}^{-1}\left(I_{1}[\underline{h}] \zeta_{x}\right)-\mu^{2} \underline{\mathfrak{I}}^{-1}\left(I_{2}[\underline{h}] \zeta_{x}\right)$,

which implies that

$$
\begin{aligned}
B_{3}+B_{4}+B_{5} & =2\left(\left[\Lambda^{s}, \underline{\mathfrak{I}}\right] \zeta_{x}, \Lambda^{s} v\right)-\left(\left[\Lambda^{s}, \mathfrak{\mathfrak { I }}\right] \underline{\mathfrak{I}}^{-1}\left(\underline{h} \zeta_{x}\right), \Lambda^{s} v\right)+\frac{2 \mu}{3}\left(\left[\Lambda^{s}, \underline{\mathfrak{I}}\right] \underline{\mathfrak{I}}^{-1} \partial_{x}\left(\underline{h}^{3} \zeta_{x x}\right), \Lambda^{s} v\right) \\
& -\frac{\mu}{\mathrm{bo}}\left(\left[\Lambda^{s}, \mathfrak{I}\right] \underline{\mathfrak{I}}^{-1}\left(\underline{h} \zeta_{x x x}\right), \Lambda^{s} v\right)+\left(\left[\Lambda^{s}, \underline{\mathfrak{h}}_{\mathrm{bo}}\right] \zeta_{x}, \Lambda^{s} v\right)+\mu^{2}\left(\left[\Lambda^{s}, I_{1}[\underline{h}]\right] \zeta_{x}, \Lambda^{s} v\right)+\mu^{2}\left(\left[\Lambda^{s}, I_{2}[\underline{h}]\right] \zeta_{x}, \Lambda^{s} v\right) \\
& =B_{31}+B_{32}+B_{33}+B_{34}+B_{35}+B_{41}+B_{51} .
\end{aligned}
$$

Using the explicit expression of $\mathfrak{I}$, the commutator estimate (38), the identities (51), and the help of Lemma 2, we get

$\left|B_{31}\right| \leq 2\left|\left(\left[\Lambda^{s}, \underline{h}\right] \zeta_{x}, \Lambda^{s} v\right)+\frac{1}{3} \mu\left(\left[\Lambda^{s}, \underline{h}^{3}\right] \zeta_{x x}, \Lambda^{s} v_{x}\right)+\frac{1}{45} \mu^{2}\left(\left[\Lambda^{s}, \underline{h}^{5}\right] \zeta_{x x x}, \Lambda^{s} v_{x x}\right)\right| \leq \varepsilon C\left(|\underline{h}-1|_{H^{s}}, \sqrt{\mathrm{bo}}\right) E^{s}(U)^{2}$.

Also, we have

$$
\begin{aligned}
\left|B_{32}\right| \leq \mid\left(\left[\Lambda^{s}, \underline{h}\right] \underline{\mathfrak{I}}^{-1}\left(\underline{h} \zeta_{x}\right), \Lambda^{s} v\right) & +\frac{1}{3} \mu\left(\left[\Lambda^{s}, \underline{h}^{3}\right] \partial_{x} \underline{\mathfrak{I}}^{-1}\left(\underline{h} \zeta_{x}\right), \Lambda^{s} v_{x}\right) \\
& +\frac{1}{45} \mu^{2}\left(\left[\Lambda^{s}, \underline{h}^{5}\right] \partial_{x}^{2} \underline{\mathfrak{I}}^{-1}\left(\underline{h} \zeta_{x}\right), \Lambda^{s} v_{x x}\right) \mid \leq \varepsilon C\left(|\underline{h}-1|_{H^{s}}, C_{s}\right) E^{s}(U)^{2},
\end{aligned}
$$

with

$$
\begin{aligned}
\left|B_{33}\right| \leq \mid \frac{2}{3} \mu\left(\left[\Lambda^{s}, \underline{h}\right] \underline{\mathfrak{J}}^{-1} \partial_{x}\left(\underline{h}^{3} \zeta_{x x}\right), \Lambda^{s} v\right)+\frac{2}{9} \mu^{2}\left(\left[\Lambda^{s}, \underline{h}^{3}\right] \partial_{x} \underline{\mathfrak{I}}^{-1} \partial_{x}\left(\underline{h}^{3} \zeta_{x x}\right), \Lambda^{s} v_{x}\right) \\
+\frac{2}{135} \mu^{3}\left(\left[\Lambda^{s}, \underline{h}^{5}\right] \partial_{x}^{2} \underline{\mathfrak{I}}^{-1} \partial_{x}\left(\underline{h}^{3} \zeta_{x x}\right), \Lambda^{s} v_{x x}\right) \mid \leq \varepsilon C\left(|\underline{h}-1|_{H^{s}}, C_{s}\right) E^{s}(U)^{2},
\end{aligned}
$$

and

$$
\begin{aligned}
\left|B_{34}\right| \leq \frac{\mu}{\mathrm{bo}} \mid\left(\left[\Lambda^{s}, \underline{h}\right] \underline{\mathfrak{I}}^{-1}\left(\underline{h} \zeta_{x x x}\right), \Lambda^{s} v\right) & +\frac{1}{3} \mu\left(\left[\Lambda^{s}, \underline{h}^{3}\right] \partial_{x} \underline{\mathfrak{I}}^{-1}\left(\underline{h} \zeta_{x x x}\right), \Lambda^{s} v_{x}\right) \\
& +\frac{1}{45} \mu^{2}\left(\left[\Lambda^{s}, \underline{h}^{5}\right] \partial_{x}^{2} \underline{\mathfrak{Y}}^{-1}\left(\underline{h} \zeta_{x x x}\right), \Lambda^{s} v_{x x}\right) \mid \leq \varepsilon C\left(|\underline{h}-1|_{H^{s}}, C_{s}\right) E^{s}(U)^{2} .
\end{aligned}
$$

For controlling $B_{35}$, one should use the explicit expression of $\mathfrak{J}_{\mathrm{bo}}$ and the fact that

$$
\left[\Lambda^{s}, M \partial_{x}^{m}\left(N \partial_{x}^{2} \cdot\right)\right] P=\left[\Lambda^{s}, M \partial_{x}^{m}\right] N \partial_{x}^{2} P+M \partial_{x}^{m}\left[\Lambda^{s}, N\right] \partial_{x}^{2} P \quad \text { with } \quad m=\{1,2\},
$$

to write the following:

$$
\begin{aligned}
B_{35}=\left(\left[\Lambda^{s}, \underline{h}\right] \zeta_{x}, \Lambda^{s} v\right)-\frac{\mu}{\mathrm{bo}}\left(\left[\Lambda^{s}, \underline{h}\right] \zeta_{x x x}, \Lambda^{s} v\right)+\frac{2}{45} \mu^{2}\left(\left[\Lambda^{s}, \underline{h}^{5}\right] \zeta_{x x x}, \partial_{x}^{2}\left(\underline{h} \Lambda^{s} v\right)\right) \\
+\frac{2}{45} \mu^{2}\left(\left[\Lambda^{s}, \underline{h} \partial_{x}^{2}\right] \underline{h^{5}} \zeta_{x x x}, \Lambda^{s} v\right)=B_{351}+B_{352}+B_{353}+B_{354},
\end{aligned}
$$

where

$$
\left|B_{351}+B_{352}+B_{353}\right| \leq \varepsilon C\left(|\underline{h}-1|_{H^{s}},|\underline{\zeta}|_{\infty}, \sqrt{\frac{\mu}{\mathrm{bo}}}\left|\underline{\zeta}_{x}\right|_{H^{s}}, \sqrt{\mathrm{bo}}\right) E^{s}(U)^{2} .
$$

For controlling $B_{354}$, one should notice the fact that

$$
\left[\Lambda^{s}, M \partial_{x}^{2}\right] N=\partial_{x}^{2}\left[\Lambda^{s}, M\right] N-2 \partial_{x}\left[\Lambda^{s}, \partial_{x} M\right] N+\left[\Lambda^{s}, \partial_{x}^{2} M\right] N=\left[\Lambda^{s}, M\right] \partial_{x}^{2} N
$$


Then, we get

$$
\left|B_{354}\right| \leq \varepsilon C\left(|\underline{h}-1|_{H^{s}}, \sqrt{\frac{\mu}{\mathrm{bo}}}\left|\underline{\zeta}_{x}\right|_{H^{s}}, \mu\left|\underline{\zeta}_{x x x}\right|_{H^{s-1}}, \sqrt{\mathrm{bo}}\right) E^{s}(U)^{2} .
$$

Therefore, we obtain

$$
\left|B_{35}\right| \leq \varepsilon C\left(|\underline{h}-1|_{H^{s}}, \underline{\zeta}_{\left.\right|_{\infty}}, \sqrt{\frac{\mu}{\mathrm{bo}}}\left|\underline{\zeta}_{x}\right|_{H^{s}}, \mu\left|\underline{\zeta}_{x x x}\right|_{H^{s-1}}, C_{s}, \sqrt{\mathrm{bo}}\right) E^{s}(U)^{2} .
$$

For controlling $B_{41}$, we use integration by parts and the commutator (38) with the help of (52), and noticing the fact that

$$
\left[\Lambda^{s}, M \partial_{x}\right] N=\partial_{x}\left[\Lambda^{s}, M\right] N-\left[\Lambda^{s}, \partial_{x} M\right] N=\left[\Lambda^{s}, M\right] \partial_{x} N,
$$

one gets

$$
\begin{aligned}
&\left|B_{41}\right| \leq \frac{4}{45} \mu^{2} \mid\left(\left[\Lambda^{s},\right.\right.\left.\left.\underline{h}^{4}\right] \zeta_{x x x}, \underline{h}_{x x} \Lambda^{s} v\right)+\left(\left[\Lambda^{s}, \underline{h}^{4}\right] \zeta_{x x x}, \underline{h}_{x} \Lambda^{s} v_{x}\right)+\left(\left[\Lambda^{s}, \underline{h}_{x}\right] \underline{h}^{4} \zeta_{x x x}, \Lambda^{s} v_{x}\right) \\
&+\left(\left[\Lambda^{s}, \underline{h}_{x x}\right] \underline{h}^{4} \zeta_{x x x}, \Lambda^{s} v\right) \mid \leq \varepsilon C\left(|\underline{h}-1|_{H^{s}}, \sqrt{\frac{\mu}{\mathrm{bo}}}\left|\underline{\zeta}_{x}\right|_{H^{s}}, \mu\left|\underline{\zeta}_{x x x}\right|_{H^{s-1}}, \sqrt{\mathrm{bo}}\right) E^{s}(U)^{2} .
\end{aligned}
$$

For $B_{51}$, we use (53) to get

$$
\left|B_{51}\right|=\frac{2}{45} \mu^{2}\left|\left(\left[\Lambda^{s}, \underline{h}^{4} \underline{h}_{x x}\right] \zeta_{x x x}, \Lambda^{s} v\right)\right| \leq \varepsilon C\left(|\underline{h}-1|_{H^{s}}, \mu\left|\underline{\zeta}_{x x x}\right|_{H^{s-1}}\right) E^{s}(U)^{2} .
$$

Thus, after collecting the information above, one gets that

$$
\left|B_{3}+B_{4}+B_{5}\right| \leq \varepsilon C\left(|\underline{h}-1|_{H^{s}},|\underline{\zeta}|_{\infty}, \sqrt{\frac{\mu}{\mathrm{bo}}}\left|\underline{\zeta}_{x}\right|_{H^{s}}, \mu\left|\underline{\zeta}_{x x x}\right|_{H^{s-1}}, C_{s}, \sqrt{\mathrm{bo}}\right) E^{s}(U)^{2} .
$$

To control $B_{6}$, we first must notice the fact that

$$
\left[\Lambda^{s}, \partial_{x}(M \cdot)\right] N=\partial_{x}\left[\Lambda^{s}, M\right] N \quad \text { and } \quad\left[\Lambda^{s}, \partial_{x}^{2}(M \cdot)\right] N=\partial_{x}^{2}\left[\Lambda^{s}, M\right] N,
$$

so one can write as in $B_{3}$ using (51) with integration by parts that

$$
\begin{aligned}
& B_{6}=\frac{1}{2 \mathrm{bo}} \varepsilon^{2} \mu^{2}\left(\left[\Lambda^{s}, \underline{h}\right] \underline{\mathfrak{I}}^{-1} \underline{h} \partial_{x}^{2}\left(\underline{\zeta}_{x}^{2} \zeta_{x}\right), \Lambda^{s} v\right)-\frac{1}{6 \mathrm{bo}} \varepsilon^{2} \mu^{3}\left(\left[\Lambda^{s}, \underline{h}^{3}\right] \partial_{x} \underline{\mathfrak{I}}^{-1} \underline{h}_{x}^{2}\left(\underline{\zeta}_{x}^{2} \zeta_{x}\right), \Lambda^{s} v_{x}\right) \\
& \left.+\frac{1}{90 \mathrm{bo}} \varepsilon^{2} \mu^{4}\left(\left[\Lambda^{s}, \underline{h^{5}}\right] \partial_{x}^{2} \underline{\mathfrak{I}}^{-1} \underline{h} \partial_{x}^{2}\left(\underline{\zeta}_{x}^{2} \zeta_{x}\right), \Lambda^{s} v_{x x}\right)-\frac{1}{2 \mathrm{bo}} \varepsilon^{2} \mu^{2}\left(\left[\Lambda^{s}, \underline{h}_{x}^{2} \underline{\zeta}_{x}^{2} \cdot\right)\right] \zeta_{x}, \Lambda^{s} v\right)=B_{61}+B_{62}+B_{63}+B_{64} .
\end{aligned}
$$

Now using Lemma 2, with the help of the Kato-Pance commutator (38), yields

$$
\left|B_{61}+B_{62}+B_{63}\right| \leq \varepsilon C\left(|\underline{h}-1|_{H^{s-1}}, C_{s}\right) \frac{\mu^{2}}{\mathrm{bo}}\left|\partial_{x}^{2}\left(\underline{\zeta}_{x}^{2} \zeta_{x}\right)\right|_{H^{s-1}} E^{s}(U) .
$$

But, we have

$$
\begin{aligned}
\frac{\mu^{2}}{\mathrm{bo}}\left|\partial_{x}^{2}\left(\underline{\zeta}_{x}^{2} \zeta_{x}\right)\right|_{H^{s-1}} \leq 2 \sqrt{\frac{\mu}{\mathrm{bo}}}\left|\underline{\zeta}_{x}\right| H^{s} \mu\left|\underline{\zeta}_{x x}\right| H^{s} \sqrt{\frac{\mu}{\mathrm{bo}}}\left|\zeta_{x}\right| H^{s} & +\frac{\mu}{\mathrm{bo}}\left|\underline{\zeta}_{x}\right|_{H^{s}}^{2} \mu\left|\zeta_{x x}\right|_{H^{s}} \\
& \leq C\left(\sqrt{\frac{\mu}{\mathrm{bo}}}\left|\underline{\zeta}_{x}\right| H^{s}, \frac{\mu}{\mathrm{bo}}\left|\underline{\zeta}_{x}\right|_{H^{s}}^{2}, \mu\left|\underline{\zeta}_{x x}\right| H^{s}\right) E^{s}(U) .
\end{aligned}
$$

Then, we get

$$
\left|B_{61}+B_{62}+B_{63}\right| \leq \varepsilon C\left(|\underline{h}-1|_{H^{s-1}}, \sqrt{\frac{\mu}{\mathrm{bo}}}\left|\underline{\zeta}_{x}\right| H^{s}, \frac{\mu}{\mathrm{bo}}\left|\underline{\zeta}_{x}\right|_{H^{s}}^{2}, \mu\left|\underline{\zeta}_{x x}\right|_{H^{s}}, C_{s}\right) E^{s}(U)^{2} .
$$

For controlling $B_{64}$, one should notice first that we have

$$
\left[\Lambda^{s}, M \partial_{x}^{2}(N \cdot)\right] P=\left[\Lambda^{s}, M \partial_{x}^{2}\right] N P+M \partial_{x}^{2}\left[\Lambda^{s}, N\right] P=\left[\Lambda^{s}, M\right] \partial_{x}^{2}(N P)+M \partial_{x}^{2}\left[\Lambda^{s}, N\right] P .
$$

Then, one gets

$$
\begin{aligned}
\left|B_{64}\right|=\frac{1}{2 \mathrm{bo}} \varepsilon^{2} \mu^{2}\left|\left(\left[\Lambda^{s}, \underline{h}\right] \partial_{x}^{2}\left(\underline{\zeta}_{x}^{2} \zeta_{x}\right), \Lambda^{s} v\right)+\left(\left[\Lambda^{s}, \underline{\zeta}_{x}^{2}\right] \zeta_{x}, \partial_{x}^{2}\left(\underline{h} \Lambda^{s} v\right)\right)\right| \\
\quad \leq \varepsilon C\left(|\underline{h}-1|_{H^{s-1}},\left|\underline{\zeta}_{x}\right|_{H^{s-1}}, \sqrt{\frac{\mu}{\mathrm{bo}}}\left|\underline{\zeta}_{x}\right| H^{s}, \frac{\mu}{\mathrm{bo}}\left|\underline{\zeta}_{x}\right|_{H^{s}}^{2}, \mu\left|\underline{\zeta}_{x x}\right|_{H^{s}}, C_{s}\right) E^{s}(U)^{2} .
\end{aligned}
$$


Therefore, we obtain

$$
\left|B_{6}\right| \leq \varepsilon C\left(|\underline{h}-1|_{H^{s-1}},\left|\underline{\zeta}_{x}\right|_{H^{s-1}}, \sqrt{\frac{\mu}{\mathrm{bo}}}\left|\underline{\zeta}_{x}\right|_{H^{s}}, \frac{\mu}{\mathrm{bo}}\left|\underline{\zeta}_{x}\right|_{H^{s}}^{2}, \mu\left|\underline{\zeta}_{x x}\right|_{H^{s}}, C_{s}\right) E^{s}(U)^{2} .
$$

To control $B_{7}$, one can write after checking the expression of $\mathfrak{I}$ and using (50) with integration by parts and the fact that $\partial_{x}\left[\Lambda^{s}, M\right] N=\left[\Lambda^{s}, M_{x}\right] N+\left[\Lambda^{s}, M\right] N_{x}$,

$$
\begin{aligned}
& \left.\left|B_{7}\right|=\varepsilon \mid\left(\left[\Lambda^{s}, \underline{v}\right] v_{x}, \underline{h} \Lambda^{s} v\right)+\frac{1}{3} \mu\left(\left[\Lambda^{s}, \underline{v}\right]\right] v_{x}, \underline{h}^{3} \Lambda^{s} v_{x}\right)+\frac{1}{3} \mu\left(\left[\Lambda^{s}, \underline{v}\right] v_{x x}, \underline{h}^{3} \Lambda^{s} v_{x}\right)+\frac{1}{45} \mu^{2}\left(\left[\Lambda^{s}, \underline{v} x x\right] v_{x}, \underline{h}^{5} \Lambda^{s} v_{x x}\right) \\
& \left.+\frac{2}{45} \mu^{2}\left(\left[\Lambda^{s}, \underline{v}\right]\right] v_{x x}, \underline{h}^{5} \Lambda^{s} v_{x x}\right)+\frac{1}{45} \mu^{2}\left(\left[\Lambda^{s}, \underline{v}\right] v_{x x x}, \underline{h}^{5} \Lambda^{s} v_{x x}\right) \mid \leq \varepsilon C\left(|\underline{h}|_{\infty},|\underline{v}|_{H^{s}},\left.\left.\sqrt{\mu}\right|_{x x}\right|_{H^{s-1}}, \mu\left|\underline{v}_{x x x}\right|_{H^{s-1}}\right) E^{s}(U)^{2} .
\end{aligned}
$$

To control $B_{8}$, one can realize $B_{3}$ and integrate by parts with the help of (51) to write

$$
\begin{aligned}
B_{8}=-\varepsilon \mu\left(\left[\Lambda^{s}, \underline{h}\right] \underline{\mathfrak{I}}^{-1} \mathcal{Q}_{1}[\underline{U}] v_{x}, \Lambda^{s} v\right)-\frac{\varepsilon \mu^{2}}{3}\left(\left[\Lambda^{s}, \underline{h}^{3}\right] \partial_{x} \underline{\mathfrak{I}}^{-1} \mathcal{Q}_{1}[\underline{U}] v_{x}, \Lambda^{s} v_{x}\right) \\
-\frac{\varepsilon \mu^{3}}{45}\left(\left[\Lambda^{s}, \underline{h}^{5}\right] \partial_{x}^{2} \underline{\mathfrak{I}}^{-1} \mathcal{Q}_{1}[\underline{U}] v_{x}, \Lambda^{s} v_{x x}\right)+\varepsilon \mu\left(\left[\Lambda^{s}, \mathcal{Q}_{1}[\underline{U}]\right] v_{x}, \Lambda^{s} v\right) .
\end{aligned}
$$

Using the expression of $\mathcal{Q}_{1}$ with the help of Lemma 2, the commutator (38), in addition to (51) and (55), one gets

$$
\left|B_{8}\right| \leq \varepsilon C\left(|\underline{h}-1|_{H^{s}},\left.\underline{v}_{x}\right|_{H^{s-1}},\left.\sqrt{\mu} \underline{v}_{x x}\right|_{H^{s-1}}, C_{s}\right) E^{s}(U)^{2} .
$$

To control $B_{9}$, one can write by integration by parts

$$
\begin{aligned}
B_{9}= & -\varepsilon \mu^{2}\left(\left[\Lambda^{s}, \underline{h}\right] \underline{\mathfrak{I}}^{-1} \mathcal{Q}_{2}[\underline{U}] v_{x}, \Lambda^{s} v\right)-\frac{\varepsilon \mu^{3}}{3}\left(\left[\Lambda^{s}, \underline{h}^{3}\right] \partial_{x} \underline{\mathfrak{I}}^{-1} \mathcal{Q}_{2}[\underline{U}] v_{x}, \Lambda^{s} v_{x}\right) \\
& -\frac{\varepsilon \mu^{4}}{45}\left(\left[\Lambda^{s}, \underline{h}^{5}\right] \partial_{x}^{2} \underline{\mathfrak{I}}^{-1} \mathcal{Q}_{2}[\underline{U}] v_{x}, \Lambda^{s} v_{x x}\right)+\varepsilon \mu^{2}\left(\left[\Lambda^{s}, \mathcal{Q}_{2}[\underline{U}]\right] v_{x}, \Lambda^{s} v\right)=B_{91}+B_{92}+B_{93}+B_{94} .
\end{aligned}
$$

Using the expression of $\mathcal{Q}_{2}$, an integration by parts with the help of Lemma 2, the commutator (38), and in addition to (51) and (55), one has

$$
\left|B_{9}\right|=\left|B_{91}+B_{92}+B_{93}+B_{94}\right| \leq \varepsilon C\left(|\underline{h}-1|_{H^{s}},\left.\sqrt{\mu} \underline{v}_{x}\right|_{H^{s}}, \mu\left|\underline{v}_{x x}\right|_{H^{s}}, C_{s}\right) E^{s}(U)^{2} .
$$

Similarly, using the expression of $\mathcal{Q}_{3}$, one can write after an integration by parts

$$
\begin{aligned}
B_{10} & =-\varepsilon \mu^{2}\left(\left[\Lambda^{s}, \underline{h}\right] \underline{\mathfrak{I}}^{-1} \mathcal{Q}_{3}[\underline{U}] v_{x}, \Lambda^{s} v\right)-\frac{\varepsilon \mu^{3}}{3}\left(\left[\Lambda^{s}, \underline{h}^{3}\right] \partial_{x} \underline{\mathfrak{J}}^{-1} \mathcal{Q}_{3}[\underline{U}] v_{x}, \Lambda^{s} v_{x}\right) \\
& -\frac{\varepsilon \mu^{4}}{45}\left(\left[\Lambda^{s}, \underline{h}^{5}\right] \partial_{x}^{2} \underline{\mathfrak{I}}^{-1} \mathcal{Q}_{3}[\underline{U}] v_{x}, \Lambda^{s} v_{x x}\right)+\varepsilon \mu^{2}\left(\left[\Lambda^{s}, \mathcal{Q}_{3}[\underline{U}]\right] v_{x}, \Lambda^{s} v\right)=B_{101}+B_{102}+B_{103}+B_{104},
\end{aligned}
$$

which can be controlled with the support of Lemma 2, the commutator (38), and in addition to (51) and (55) by the following:

$$
\varepsilon C\left(|\underline{h}-1|_{H^{s}},\left.\sqrt{\mu} \underline{v}_{x x}\right|_{H^{s-1}},\left.\mu \underline{v}_{x x}\right|_{H^{s}}, C_{s}\right) E^{s}(U)^{2} .
$$

Eventually, as a conclusion, one gets

$$
\begin{aligned}
\left|\left(\left[\Lambda^{s}, A[\underline{U}]\right] \partial_{x} U, S \Lambda^{s} U\right)\right| \leq \varepsilon C\left(|\underline{h}-1|_{H^{s}},|\underline{\zeta}|_{H^{s}}, \sqrt{\frac{\mu}{\mathrm{bo}}}\left|\underline{\zeta}_{x}\right| H^{s}, \frac{\mu}{\mathrm{bo}}\left|\underline{\zeta}_{x}\right|_{H^{s}}^{2},\right. \\
\\
\left.\mu\left|\underline{\zeta}_{x x}\right| H^{s},|\underline{v}|_{H^{s}},\left.\sqrt{\mu} \underline{v}_{x}\right|_{H^{s}},\left.\mu \underline{v}_{x x}\right|_{H^{s}}, C_{s}, \text { bo }\right) E^{s}(U)^{2} .
\end{aligned}
$$

- Estimation of $\left(\Lambda^{s} \zeta,\left[\partial_{t}, \underline{\mathfrak{J}}_{\mathrm{bo}}\right] \Lambda^{s} \zeta\right)$. One can write, after checking the expression of $\underline{\mathfrak{J}}_{\mathrm{bo}}$ and doing an integration by parts, that

$$
\left|\left(\Lambda^{s} \zeta,\left[\partial_{t}, \underline{\mathfrak{J}}_{\mathrm{bo}}\right] \Lambda^{s} \zeta\right)\right|=\left|\frac{2}{45} \mu^{2}\left(\partial_{t} \underline{h}^{4} \Lambda^{s} \zeta_{x x}, \Lambda^{s} \zeta_{x x}\right)\right| \leq \varepsilon C\left(\left|\partial_{t} \underline{h}\right|_{\infty}\right) E^{s}(U)^{2} .
$$

- Estimation of $\left(\Lambda^{s} v,\left[\partial_{t}, \mathfrak{I}\right] \Lambda^{s} v\right)$. Remarking that we have $\left[\partial_{t}, \underline{h}\right] \Lambda^{s} v=\partial_{t} \underline{h} \Lambda^{s} v \quad\left[\partial_{t}, \partial_{x}\left(\underline{h}^{3} \partial_{x} \cdot\right)\right] \Lambda^{s} v=\partial_{x}\left(\partial_{t} \underline{h}^{3} \Lambda^{s} v_{x}\right), \quad$ and $\quad\left[\partial_{t}, \partial_{x}^{2}\left(\underline{h}^{5} \partial_{x}^{2}\right)\right] \Lambda^{s} v=\partial_{x}^{2}\left(\partial_{t} \underline{h}^{5} \Lambda^{s} v_{x x}\right)$, 
one gets using integration by parts

$$
\begin{aligned}
\left|\left(\Lambda^{s} v,\left[\partial_{t}, \underline{\mathfrak{I}}\right] \Lambda^{s} v\right)\right|=\mid\left(\partial_{t} \underline{h} \Lambda^{s} v, \Lambda^{s} v\right)+ & \frac{\mu}{3}\left(\partial_{t} \underline{h}^{3} \Lambda^{s} v_{x}, \Lambda^{s} v_{x}\right) \\
& +\frac{\mu^{2}}{45}\left(\partial_{t} \underline{h}^{5} \Lambda^{s} v_{x x}, \Lambda^{s} v_{x x}\right) \mid \leq \varepsilon C\left(\left|\partial_{t} \underline{h}\right|_{\infty}, E^{s}(\underline{U})\right) E^{s}(U)^{2} .
\end{aligned}
$$

Gathering the information provided by the above estimates and using the fact that $H^{s}(\mathbb{R})$ is continuously embedded in $W^{1, \infty}(\mathbb{R})$, we get

$$
\frac{1}{2} e^{\varepsilon \lambda t} \partial_{t}\left(e^{-\varepsilon \lambda t} E^{s}(U)^{2}\right) \leq \varepsilon\left(C\left(E^{s}(\underline{U}),\left|\partial_{t} \underline{h}\right|_{L^{\infty}}\right)-\lambda\right) E^{s}(U)^{2} .
$$

Taking $\lambda=\lambda_{T}$ large enough (how large depending on $\sup _{t \in\left[0, \frac{T}{\varepsilon}\right]} C\left(E^{s}(\underline{U}),\left|\partial_{t} \underline{h}\right|_{L^{\infty}}\right)$ to have the first term of the right-hand side negative for all $t \in\left[0, \frac{T}{\varepsilon}\right]$, one deduces

$$
\forall t \in\left[0, \frac{T}{\varepsilon}\right], \quad \frac{1}{2} e^{\varepsilon \lambda t} \partial_{t}\left(e^{-\varepsilon \lambda t} E^{s}(U)^{2}\right) \leq 0 .
$$

Integrating this differential inequality with the help of Grönwall's inequality therefore yields

$$
\forall t \in\left[0, \frac{T}{\varepsilon}\right], \quad E^{s}(U(t)) \leq\left(e^{\varepsilon \lambda_{T} t}\right)^{1 / 2} E^{s}\left(U_{0}\right),
$$

which is the desired result. The existence and uniqueness of the solution is a direct adaptation of the proof in Appendix A of Ref. 2.

\section{Main result}

Our goal in this paper, which is the well-posedness in $X^{s}=H^{s+2}(\mathbb{R}) \times H^{s+2}(\mathbb{R})$ for some $s>\frac{3}{2}$, of the extended system (29) with surface tension for a finite large time existence $t=O\left(\frac{1}{\varepsilon}\right)$ is proved by the following theorem.

Theorem 1. Let $t_{0}>\frac{1}{2}$ and $s \geq t_{0}+1$. Let also $U_{0}=\left(\zeta_{0}, v_{0}\right)^{T} \in X^{s}$ be such that (33) is satisfied. Then there exists a maximal $T_{\max }>0$, uniformly bounded from below with respect to $\varepsilon, \mu \in(0,1)$, such that the extended one-dimensional Green-Naghdi equations (29) with surface tension admit a

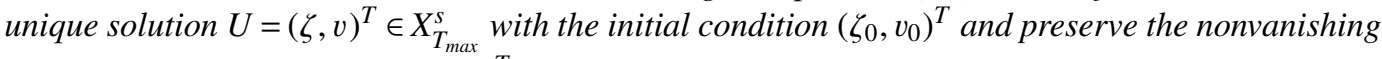
depth condition (33) for any $t \in\left[0, \frac{T_{\max }}{\varepsilon}\right)$. In particular, if $T_{\max }<\infty$, one has

$$
|U(t, \cdot)|_{X^{s}} \longrightarrow \infty \quad \text { as } \quad t \longrightarrow \frac{T_{\max }}{\varepsilon}
$$

or

$$
\inf _{\mathbb{R}} h(t, \cdot)=\inf _{\mathbb{R}} 1+\varepsilon \zeta(t, \cdot) \longrightarrow 0 \quad \text { as } \quad t \longrightarrow \frac{T_{\max }}{\varepsilon} .
$$

Proof. The proof is a straightforward readjustment of the proof of Theorem 7.3 in Ref. 24 using the energy estimate proved in Proposition 1, which is itself an adaptation of the proof of the wellposedness of hyperbolic systems (see Ref. 19 for general details). Here is a brief sketch of the proof commenced in considering an iterative scheme of approximate solution $\left(U^{n}=\left(\zeta^{n}, v^{n}\right)\right)_{n \geq 0}$ through the induction relation

$$
\forall n \in \mathbb{N}, \quad\left\{\begin{array}{l}
\partial_{t} U^{n+1}+A\left[U^{n}\right] \partial_{x} U^{n+1}=0 \\
U_{l_{t=0}^{n+1}=U_{0}} \quad \text { with } \quad U^{0}=U_{0}
\end{array} .\right.
$$

By Proposition 1, we know that there exists a unique solution $U^{n+1} \in C\left([0, \infty), X^{s}\right)$ to system (57) and $U^{n}$ satisfies the depth-condition. Let $R>0$ be such that $E^{s}\left(U^{0}\right) \leq \frac{R}{2}$. It follows from Proposition 1 that $U^{n+1}$ satisfies the following inequality:

$$
E^{s}\left(U^{n+1}\right)(t) \leq\left(e^{\varepsilon \lambda_{T} t}\right)^{1 / 2} E^{s}\left(U^{0}\right) .
$$


Hence by induction on $n$ and the uniform equivalence between $|\cdot| X^{s}$ and $E^{s}\left(U^{n+1}\right)$, we get

$$
\sup _{t \in\left[0, \frac{T}{\varepsilon}\right]}\left|U^{n+1}(t)\right|_{X^{s}} \leq C\left(\frac{1}{h_{\min }}\right) R .
$$

Now, using (57) we have $\partial_{t} \zeta^{n+1}+\varepsilon v^{n} \zeta_{x}^{n+1}+v_{x}^{n+1}+\varepsilon \zeta^{n} v_{x}^{n+1}=0$, and then we deduce that

$$
\left|\partial_{t} h^{n+1}\right|_{\infty}=\varepsilon\left|\partial_{t} \zeta^{n+1}\right|_{\infty} \leq \varepsilon C\left(\frac{1}{h_{\min }}\right) R .
$$

Therefore, one attains $\lambda_{T}$ independent of $\partial_{t} U^{n}$. Moreover, we have $h_{\mid t=0}^{n+1} \geq h_{\min }$. Consequently, one can deduce that

$$
h^{n+1}=h_{\mid t=0}^{n+1}+\varepsilon \int_{0}^{t} \partial_{t} \zeta^{n+1} \geq h_{\min }-\varepsilon T C\left(\frac{1}{h_{\min }}\right) R .
$$

Therefore, to $U^{n+1}$ satisfy (33), it is possible to choose $T$ small enough, for example, $T=\frac{1}{C\left(\frac{1}{h_{\min }}\right) R}$. $\frac{h_{\min }}{2}$. Thus $U^{n+1}$ satisfies (33) on $\left[0, \frac{T}{\varepsilon}\right)$ with $h_{\min }$ replaced by $\frac{h_{\min }}{2}$.

Finally, we deduce that the Cauchy problem (57) has a unique solution $U^{n+1}$ satisfying (33) and the inequality (58) when $0 \leq t \leq \frac{T}{\varepsilon}$ and $\lambda_{T}$ depending only on $\sup _{\left[0, \frac{T}{\varepsilon}\right]} E^{s}\left(U^{n}\right)$. Thanks to this energy estimate (58), one can deduce that $U^{n}$ is uniformly bounded in $X^{s}$. Now, in order to prove the convergence of $U^{n}$ toward a solution to our nonlinear problem (40), one should define $V^{n}$ such that $U^{n}=U^{0}+\sum_{0}^{n-1} V^{n}$. Then, $V^{n}=U^{n+1}-U^{n}$ satisfies the system

$$
\forall n \in \mathbb{N}, \quad\left\{\begin{array}{l}
\partial_{t} V^{n}+A\left[U^{n}\right] \partial_{x} V^{n}=-\left(A\left[U^{n}\right]-A\left[U^{n-1}\right]\right) \partial_{x} U^{n}, \\
V_{l_{t=0}}^{n}=0 .
\end{array}\right.
$$

We use now the symmetry property of $S^{n}=\left(\begin{array}{cc}\mathfrak{J}_{\text {bo }}^{n} & 0 \\ 0 & \mathfrak{J}^{n}\end{array}\right)$, as well as the definition (45) of $E^{s}(U)$, so one may write

$$
\frac{1}{2} \frac{d}{d t}\left(E^{0}\left(V^{n}\right)\right)^{2}=-\left(S^{n} A\left[U^{n}\right] \partial_{x} V^{n}, V^{n}\right)+\frac{1}{2}\left(\left[\partial_{t}, S^{n}\right] V^{n}, V^{n}\right)-\left(S^{n}\left(A\left[U^{n}\right]-A\left[U^{n-1}\right]\right) \partial_{x} U^{n}, V^{n}\right),
$$

where $U^{n}=\left(\zeta^{n}, v^{n}\right)^{T}, h^{n}=1+\varepsilon \zeta^{n}, \mathfrak{J}^{n}=h^{n}+\mu \mathcal{T}\left[h^{n}\right]-\mu^{2} \mathfrak{T}\left[h^{n}\right]$, and $\mathfrak{J}_{\text {bo }}^{n}=1-\frac{\mu}{\text { bo }} \partial_{x}^{2}(\cdot)+$ $\frac{2}{45} \mu^{2} \partial_{x}^{2}\left(\left(h^{n}\right)^{4} \partial_{x}^{2} \cdot\right)$ for all $n \geq 0$. Now we estimate each component on the rhs of (63) as in Proposition 1 and noticing (60), so one can deduce

$$
\begin{aligned}
\frac{1}{2} \frac{d}{d t}\left(E^{0}\left(V^{n}\right)\right)^{2} & \leq \varepsilon C\left(E^{s}\left(U^{n}\right), \sqrt{\mathrm{bo}}\right) E^{0}\left(V^{n}\right)^{2}+\varepsilon C\left(E^{s}\left(U^{n-1}\right), E^{s}\left(U^{n}\right), \sqrt{\mathrm{bo}}\right) E^{0}\left(V^{n}\right) E^{0}\left(U^{n}-U^{n-1}\right) \\
& \leq \varepsilon C_{0}\left[E^{0}\left(V^{n}\right)^{2}+E^{0}\left(V^{n}\right) E^{0}\left(U^{n}-U^{n-1}\right)\right],
\end{aligned}
$$

where by (58) we have $C_{0}=C\left(E^{0}\left(U^{0}\right)(0), \sqrt{\mathrm{bo}}\right)$ independent of $n$ and $t$. Applying Grönwall's inequality therefore yields to have

$$
\forall t \in\left[0, \frac{T}{\varepsilon}\right], \quad E^{0}\left(V^{n}(t)\right) \leq \varepsilon C_{0} \int_{0}^{t} e^{\varepsilon \lambda_{T}\left(t-t_{1}\right)} E^{0}\left(V^{n-1}\right)\left(t_{1}\right) d t_{1} \leq \varepsilon C_{*} \int_{0}^{t} E^{0}\left(V^{n-1}\right)\left(t_{1}\right) d t_{1} .
$$

Hence, when $0 \leq t \leq T / \varepsilon$ and $C_{*}$ independent of $n$ and $t$, we have

$$
E^{0}\left(V^{n}(t)\right) \leq \varepsilon^{n} C_{*}^{n}\left\{\int_{0}^{t} \int_{0}^{t_{1}} \ldots \int_{0}^{t_{n-1}} d t_{n} d t_{n-1} \ldots d t_{1}\right\} \sup _{t_{1} \in\left[0, \frac{T}{\varepsilon}\right]} E^{0}\left(V^{0}\right)\left(t_{1}\right)=\frac{\varepsilon^{n} C_{*}^{n} t^{n}}{n !} \sup _{t_{1} \in\left[0, \frac{T}{\varepsilon}\right]} E^{0}\left(V^{0}\right)\left(t_{1}\right) .
$$

Finally, one can conclude classically (see Ref. 19 for general details and Ref. 24 for special models) the existence of $T_{\max }=T\left(E^{s}\left(U_{0}\right)\right)>0$ and of a unique solution $U \in X_{T_{\max }^{s}}$ to (29) preserving the depth-condition (33) for any $t \in\left[0, \frac{T_{\max }}{\varepsilon}\right]$ as a limit of the iterative scheme (57).

The fact that $T_{\max }$ is bounded from below by some $T>0$ independent of $\varepsilon, \mu \in(0,1)^{2}$ follows from the analysis above, while the behavior of the solution as $t \rightarrow T_{\max }$ if $T_{\max }<\infty$ follows from standard continuation arguments. 


\section{ACKNOWLEDGMENTS}

The authors gratefully acknowledge the partially support from the Lebanese University research program.

${ }^{1}$ D. Lannes. The Water Waves Problem: Mathematical Analysis and Asymptotics, Volume 188 of Mathematical Surveys and Monographs (American Mathematical Society, Providence, RI, 2013).

2 I. Samer, "Large time existence for 1D Green-Naghdi equations," Nonlinear Anal.: Theory, Methods Appl. 74, 81-93 (2011).

${ }^{3}$ I. Samer, "Derivation and analysis of a new 2D Green-Naghdi system," Nonlinearity 23, 2889-2904 (2010).

${ }^{4} \mathrm{~L}$. V. Ovsjannikov, "Cauchy problem in a scale of Banach spaces and its application to the shallow water theory justification," in Application of Methods of Functional Analysis to Problems of Mechanics (IUTAM/IMU-Symposium, Marseille, 1975), Lecture Notes in Mathematics (Springer, 1976), Vol. 503, pp. 426-437.

${ }^{5}$ N. Makarenko, "A second long-wave approximation in the Cauchy-Poisson problem," Dyn. Contin. Media 77, 56-72 (1986).

${ }^{6}$ Y. Matsuno, "Hamiltonian formulation of the extended Green-Naghdi equations," Phys. D 301-302, 1-7 (2015).

${ }^{7}$ Y. Matsuno, "Hamiltonian structure for two-dimentional extended Green-Naghdi equations," Proc. R. Soc. A 472, 20160127 (2016).

${ }^{8}$ T. Iguchi, "A shallow water approximation for water waves,” J. Math. Kyoto Univ. 49, 13-55 (2009).

${ }^{9}$ V. E. Zakharov, "Stability of periodic waves of finite amplitude on the surface of a deep fluid," J. Appl. Mech. Techn. Phys. 9, 190-194 (1968).

${ }^{10}$ Y. A. Li, "A shallow-water approximation to the full water wave problem," Commun. Pure Appl. Math. 59, 1225-1285 (2006).

${ }^{11}$ A. E. Green, N. Laws, and P. M. Naghdi, "On the theory of water waves," Proc. R. Soc. A 338, 43-55 (1974).

${ }^{12}$ A. E. Green and P. M. Naghdi, "A derivation of equations for wave propagation in water of variable depth,” J. Fluid Mech. 78, 237-246 (1976).

${ }^{13}$ B. Alvarez-Samaniego and D. Lannes, "Large time existence for 3D water-waves and asymptotics," Invent. Math. 171, 485-541 (2008).

${ }^{14}$ B. Alvarez-Samaniego and D. Lannes, "A Nash-Moser theorem for singular evolution equations. Application to the Serre and Green-Naghdi equations,” Indiana Univ. Math. J. 57, 97-131 (2008).

${ }^{15}$ D. Lannes and P. Bonneton, "Derivation of asymptotic two-dimensional time-dependent equations for surface water wave propagation," Phys. Fluids 21, 016601 (2009).

${ }^{16}$ D. M. Ambrose and N. Masmoudi, "The zero surface tension limit two-dimentional water waves," Commun. Pure Appl. Math. 58, 1287-1315 (2005).

${ }^{17}$ D. M. Ambrose and N. Masmoudi, “The zero surface tension limit three-dimentional water waves,” Indiana Univ. Math. J. 58, 479-521 (2009)

${ }^{18}$ M. Ming and Z. Zhang, "Well-posedness of the water-wave problem with surface tension," J. Math. Pures Appl. 92, 429-455 (2009).

${ }^{19}$ S. Alinhac and P. Gérard, Opérateurs pseudo-différentiels et théorème de Nash-Moser, Savoirs Actuels (InterEditions, Paris; Editions du Centre National de la Recherche Scientifique (CNRS), Meudon, 1991), 190 pp.

${ }^{20}$ S. V. Bazdenkov, N. N. Morozkov, and O. P. Pogutse, "Dispersive effects in two-dimentional hydrodynamics," Sov.Phys. Dokl. 32, 262-264 (1987).

${ }^{21}$ T. Kano and T. Nishida, "Sur les ondes de surface de l'eau avec une justification mathématique des équations des ondes en eau peu profonde," J. Math. Kyoto Univ. 19, 335-370 (1979).

${ }^{22}$ G. Wei, J. T. Kirby, S. T. Grilli, and R. Subramanya, "A fully nonlinear Boussinesq model for surface waves. I. Highly nonlinear unsteady waves," J. Fluid Mech. 294, 71-92 (1995).

${ }^{23}$ T. B. Benjamin, J. L. Bona, and J. J. Mahony, "Model equations for long waves in nonlinear dispersive systems," Philos. Trans. R. Soc., A 272(1220), 47-78 (1972).

${ }^{24} \mathrm{~V}$. Duchene, S. Israwi, and R. Talhouk, "A new fully justified asymptotic model for the propagation of internal waves in the Camassa-Holm regime,” SIAM J. Math. Anal. 47(1), 240-290 (2015). 